\title{
Rectifiability and approximate differentiability of higher order for sets
}

\author{
Mario Santilli
}

October 11, 2018

\begin{abstract}
The main goal of this paper is to develop a concept of approximate differentiability of higher order for subsets of the Euclidean space that allows to characterize higher order rectifiable sets, extending somehow well known facts for functions. We emphasize that for every subset $A$ of the Euclidean space and for every integer $k \geq 2$ we introduce the approximate differential of order $k$ of $A$ and we prove it is a Borel map whose domain is a (possibly empty) Borel set. This concept could be helpful to deal with higher order rectifiable sets in applications.
\end{abstract}

MSC-classes 2010. 28A75 (Primary); 49Q15 (Secondary).

Keywords. Higher-order rectifiability, approximate differentiability, Borel functions.

\section{Introduction}

For notation and terminology, see the dedicated section in the Introduction.

Motivation. This paper deals with the class of subsets of the $n$ dimensional Euclidean space that can be covered, up to a set of $\mathscr{H}^{m}$ measure zero, by countably many $m$ dimensional submanifolds of class $k$, for some integer $k \geq 1$. These sets are called countably $\left(\mathscr{H}^{m}, m\right)$ rectifiable of class $k$; see the definition of Higher order rectifiability later in this section. Evidently, this class of sets finds applications in the study of differentiability properties of "singular submanifolds" in geometry and analysis. In what follows, we mention some of these applications.

In Calculus of Variations, solutions of problems involving elliptic functionals can be efficiently modeled using the class of varifolds, originally introduced by Almgren in the 60's (the classical reference is [All72]). Even just considering the class of integral varifolds $V$ in $\mathbf{R}^{n}$ whose first variation with respect to area is represented by integrating a function 11 in $\mathbf{L}_{\infty}^{\text {loc }}\left(\|V\|, \mathbf{R}^{n}\right)$ (usually called mean curvature), it is well known that there are examples whose support does not locally correspond to a graph of a function of class 1 , on a set of positive $\mathscr{H}^{m}$ measure (see All72, 8.1(2)] for a 1 dimensional example). However, combining the recent result in Men13 with [All72, 8.3], we can deduce that the support of an integral varifold $V$ with mean curvature in $\mathbf{L}_{m}^{\text {loc }}\left(\|V\|, \mathbf{R}^{n}\right)$ is countably

\footnotetext{
${ }^{1}$ See [Fed69, 2.4.12] for the definition of Lebesgue spaces.
} 
$\left(\mathscr{H}^{m}, m\right)$ rectifiable of class 2 . Yet, it is unknown if "class 2" in the previous assertion can be replaced by "class $k$, for every integer $k \geq 1$ ", in case the first variation of $V$ with respect to area equals zero.

The level sets of maps between Euclidean spaces with distributional derivatives up to order $k$ representable by integration can be covered, up to a set of $\mathscr{H}^{m}$ measure zero, by countably many submanifolds of class $k$ of the appropriate dimension, see [BHS05, 1.6].

In Convex Geometry, Alb94, Theorem 3] asserts that if for a convex subset of the $n$ dimensional Euclidean space we define the $m$-th stratum as the set of points where the normal cone has dimension at least $n-m$, then the $m$ th stratum is countably $\left(\mathscr{H}^{m}, m\right)$ rectifiable of class 2 . This result has been recently extended to every closed subsets of Euclidean space in MS17.

Finally we mention the work in [Del12] in the context of Legendrian currents and the work in Kol16 in relation to the notion of discrete curvatures for sets.

Results of the present paper. The main contribution of this paper is to introduce a notion of approximate differentiability of higher order for subsets of the Euclidean space and to use it in order to characterize higher order rectifiable sets. For functions whose domain is a subset of the Euclidean space this is a well known fact, established in [Whi51, Fed69, § 3.1] and [sa87. More specifically these results can be combined to get the following result, see 2.11 and 2.12 .

1.1 Theorem (Federer, Isakov, Whitney). If $1 \leq m<n$ and $k \geq 1$ are integers, $0 \leq \alpha \leq 1, A \subseteq \mathbf{R}^{m}$ is $\mathscr{L}^{m}$ measurable and $f: A \rightarrow \mathbf{R}^{n-m}$ is $\mathscr{L}^{m}\llcorner A$ measurable, then $f$ is approximately differentiable of order $(k, \alpha)$ at $\mathscr{L}^{m}$ a.e. $a \in A$ if and only if there exist countably many functions $g_{j}: \mathbf{R}^{m} \rightarrow \mathbf{R}^{n-m}$ of class $(k, \alpha)$ such that

$$
\mathscr{L}^{m}\left(A \sim \bigcup_{j=1}^{\infty}\left\{x: g_{j}(x)=f(x)\right\}\right)=0
$$

In this paper we establish this result for subsets of Euclidean space. In fact, employing the notion of approximate differentiability of higher order for sets introduced in 3.8 we can prove, in 3.23 and 5.6 , the following result.

1.2 Theorem. If $1 \leq m \leq n$ and $k \geq 1$ are integers, $0 \leq \alpha \leq 1, A \subseteq \mathbf{R}^{n}$ is $\mathscr{H}^{m}$ measurable and $\mathscr{H}^{m}(A)<\infty$, then $A$ is approximately differentiable of order $(k, \alpha)$ at $\mathscr{H}^{m}$ a.e. $a \in A$ if and only if $A$ is $\left(\mathscr{H}^{m}, m\right)$ rectifiable of class $(k, \alpha)$.

It is worth to compare this result with other results in the literature. Firstly, this result can be seen as a generalization to the case of higher order rectifiability of the well known fact in Geometric Measure Theory that $\left(\mathscr{H}^{m}, m\right)$ rectifiable set: 3 of class 1 can be characterized among all the $\mathscr{H}^{m}$ measurable subsets of $\mathbf{R}^{n}$ with finite $\mathscr{H}^{m}$ measure through the existence of an $m$ dimensional "measure theoretic tangent space" at $\mathscr{H}^{m}$ a.e. points of the set. There are essentially two natural ways to define this notion of measure theoretic tangency. One uses a blow up procedure and the other one uses densities of Hausdorff measures.

\footnotetext{
${ }^{2}$ For the definition of approximate differentiability for functions, see 2.3

${ }^{3}$ By Fed69, 3.2.29] the notion of rectifiability of class 1 coincides with the classical notion of rectifiability phrased in terms of images of Lipschitzian maps, see [Fed69, 3.2.14].
} 
1.3 Definition (Simor 4 ). Suppose $1 \leq m \leq n$ are integers, $A \subseteq \mathbf{R}^{n}$ and $a \in \mathbf{R}^{n}$. An $m$ dimensional plane $T \in \mathbf{G}(n, m)$ is the $m$ dimensional approximate tangent plane of $A$ at $a$ if and only if there exists $0<\theta<\infty$ such that

$$
\lim _{r \rightarrow 0+} r^{-m} \int_{A} f((x-a) / r) d \mathscr{H}^{m} x=\theta \int_{T} f d \mathscr{H}^{m} \quad \text { whenever } f \in \mathscr{K}\left(\mathbf{R}^{n}\right) .
$$

1.4 Definition (Federen 5 ). Suppose $1 \leq m \leq n$ are integers, $A \subseteq \mathbf{R}^{n}$ and $a \in \mathbf{R}^{n}$. A vector $v \in \mathbf{R}^{n}$ is an $\left(\mathscr{H}^{m}\llcorner A, m)\right.$ approximate tangent vector at $a$ if and only if $\boldsymbol{\Theta}^{* m}\left(\mathscr{H}^{m}\llcorner A \cap \mathbf{E}(a, v, \epsilon), a)>0\right.$ for every $\epsilon>0$. An $m$ dimensional plane $T \in \mathbf{G}(n, m)$ is the $m$ dimensional approximate tangent plane of $A$ at $a$ if and only if $T$ equals the set of all $\left(\mathscr{H}^{m}\llcorner A, m)\right.$ approximate tangent vectors at $a$.

In 1.3 and in 1.4 it is not difficult to see that $m$ and $T$ are uniquely determined by $A$ and $a$. Either employing the notion in 1.3 or the one in 1.4 the following well known result holds.

1.5 Theorem (Federe16, Simon7). Suppose $1 \leq m \leq n$ are integers and $A \subseteq$ $\mathbf{R}^{n}$ is $\mathscr{H}^{m}$ measurable with $\mathscr{H}^{m}(A)<\infty$. Then $A$ is $\left(\mathscr{H}^{m}, m\right)$ rectifiable of class 1 if and only if $A$ admits the $m$ dimensional approximate tangent plane at $\mathscr{H}^{m}$ a.e. $a \in A$.

Suppose now $A \subseteq \mathbf{R}^{n}$ and $a \in \mathbf{R}^{n}$. The definition of approximate differentiability of order 1 introduced in 3.8 (see also 3.19) is equivalent to require the existence of a measure theoretic tangent space at $a$, denoted by ap $\operatorname{Tan}(A, a)$. If $A$ is $\mathscr{H}^{m}$ measurable and $\mathscr{H}^{m}(A)<\infty$, the sets of points of $A$ where the aforementioned approximate tangent spaces are $m$ dimensional subspaces, coincide up to a set of $\mathscr{H}^{m}$ measure zero (notice 3.21, 3.14, 3.4 and 3.17).

The problem of generalizing [1.5 to the case of higher order rectifiability was addressed in AS94. In that paper a notion of differentiability of order 2 and order $(1, \alpha)$ for every $0<\alpha \leq 1$, is introduced by means of a blow up procedure similar to 1.3. However, as it is pointed out in [AS94. pp. 7-8], examples show that $\left(\mathscr{H}^{m}, m\right)$ rectifiable sets of class 2 may fail to be differentiable of order 2 in the sense of [AS94] at $\mathscr{H}^{m}$ a.e. points. Therefore, in order to generalize 1.5, additional technical hypotheses on the structure of the sets are needed in the main theorems [AS94, 3.5, 3.12]. These facts suggest the possibility to consider a different notion of (approximate) differentiability of order greater than 1 and in the present paper we accomplish such goal. Our notion is based on the approach of 1.4 (see also 3.9), rather than 1.3 ,

For every integer $k \geq 2$, the notion of approximate differentiability of order $k$ for a subset $A \subseteq \mathbf{R}^{n}$ naturally induces a notion of approximate differential of order $k$, ap $\mathrm{D}^{k} A$, of $A$; see 3.20 For every $A \subseteq \mathbf{R}^{n}$, this is always a Borel map with values in $\bigodot^{k}\left(\mathbf{R}^{n}, \mathbf{R}^{n}\right)$ whose domain is a (possibly empty) Borel subset of $\mathbf{R}^{n}$, see 5.5. Moreover the approximate differential of order 2 naturally induces a notion of "approximate second fundamental form". In fact, for every $a \in \mathrm{dmn}$ ap $\mathrm{D}^{2} A$ this can be defined as the symmetric bilinear form

$$
\text { ap } \mathrm{D}^{2} A(a) \mid \operatorname{ap} \operatorname{Tan}(A, a) \times \text { ap } \operatorname{Tan}(A, a) .
$$

\footnotetext{
${ }^{4}$ See [Sim83, 11.2, 11.4] and [FM99, 2.2].

${ }^{5}$ See Fed69, 3.2.16].

${ }^{6}$ See Fed69, 3.2.19, 3.3.17]

${ }^{7}$ See [Sim83, 11.6, 11.8].
} 
In 3.25 and 4.12 two classical properties of the second fundamental form of submanifolds of class 2 are extended to our setting.

Finally we mention that a notion of pointwise differentiability for subsets of the Euclidean space has been recently developed in [Men16 to study higher order differentiability properties of stationary varifolds. In the present paper we establish the connection between the notion of approximate differentiability and pointwise differentiability in 4.11

Notation and basic definitions. The notation and the terminology used withouth comments agree with [Fed69, pp. 669-676]. However, for the reader's convenience, sometimes we use footnotes to point out the references in Fed69. Moreover we add the following classical definitions.

Distance function from a set. Let $A \subseteq \mathbf{R}^{n}$. We define $\boldsymbol{\delta}_{A}$ to be the function on $\mathbf{R}^{n}$ such that $\boldsymbol{\delta}_{A}(x)=\inf \{|x-a|: a \in A\}$ for $x \in \mathbf{R}^{n}$.

Orthogonal projections. If $1 \leq m \leq n$ are integers we define $\mathbf{G}(n, m)$ to be the set of all $m$ dimensional subspaces of $\mathbf{R}^{n}$. If $T \in \mathbf{G}(n, m)$ we define $T_{\natural}: \mathbf{R}^{n} \rightarrow \mathbf{R}^{n}$ to be the linear map such that

$$
T_{\natural}^{*}=T_{\natural}, \quad T_{\natural} \circ T_{\natural}=T_{\natural}, \quad \operatorname{im} T_{\natural}=T,
$$

and we define $T^{\perp}=\operatorname{ker} T_{\mathrm{\natural}}$.

Pointwise differentiability for functions. Suppose $X$ and $Y$ are normed vector spaces, $k \geq 0$ is an integer, $0 \leq \alpha \leq 1, g$ maps a subset of $X$ into $Y$ and $a \in X$. We say that $g$ is pointwise differentiable of order $(k, \alpha)$ at $a$ if and only if there exists an open set $U \subseteq X$ and a polynomial function $P: X \rightarrow Y$ of degree at most $k$ such that $a \in \bar{U} \subseteq \operatorname{dmn} g, g(a)=P(a)$,

$$
\lim _{x \rightarrow a} \frac{|g(x)-P(x)|}{|x-a|^{k}}=0 \text { if } \alpha=0, \quad \limsup _{x \rightarrow a} \frac{|g(x)-P(x)|}{|x-a|^{k+\alpha}}<\infty \text { if } \alpha>0 .
$$

In this case $P$ is unique and the pointwise differentials of order $i$ of $f$ at $a$ are defined by $\operatorname{pt~}^{i} g(a)=\mathrm{D}^{i} P(a)$ for $i=0, \ldots, k$.

Functions and submanifolds of class $(k, \alpha)$. Suppose $X$ and $Y$ are normed vector spaces, $k \geq 0$ is an integer, $0 \leq \alpha \leq 1, g$ maps some open subset of $X$ into $Y$ and $a \in X$. We say that $g$ is of class $(k, \alpha)$ if and only if $g$ is of class $k$ and each point of dmn $g$ has an open neighbourhood $U$ such that $\left(\mathrm{D}^{k} f\right) \mid U$ satisfies a Hölder condition with exponent $\alpha$.

Suppose $k \geq 0$ is an integer and $0 \leq \alpha \leq 1$. The notion of diffeomorphism of class $(k, \alpha)$ is made by replacing "class $k$ " with "class $(k, \alpha)$ " in [Fed69, 3.1.18]. Analogously the notion of $\mu$ dimensional submanifold of class $(k, \alpha)$ of $\mathbf{R}^{n}$ is made by replacing "class $k$ " with "class $(k, \alpha)$ " in [Fed69, 3.1.19].

Second fundamental form. If $1 \leq m \leq n$ are integers, $M$ is an $m$ dimensional submanifold of class 2 of $\mathbf{R}^{n}$ and $a \in M$ then we call second fundamental form of $M$ at $a$ the unique symmetric 2 linear function

$$
\mathbf{b}_{M}(a): \operatorname{Tan}(M, a) \times \operatorname{Tan}(M, a) \rightarrow \operatorname{Nor}(M, a)
$$

such that $\mathbf{b}_{M}(a)(u, v) \bullet \nu(a)=-\mathrm{D} \nu(a)(u) \bullet v$ for each $u, v \in \operatorname{Tan}(M, a)$, whenever $\nu: M \rightarrow \mathbf{R}^{n}$ is of class 1 relative to $M$ with $\nu(x) \in \operatorname{Nor}(M, x)$ for every $x \in M$. 
Cones. A subset $C \subseteq \mathbf{R}^{n}$ is called cone if and only if $\lambda x \in C$ whenever $x \in C$ and $\lambda>0$.

Higher order rectifiability 8 . Suppose $1 \leq m \leq n$ are integers and $\phi$ is a measure over $\mathbf{R}^{n}$. A subset $A \subseteq \mathbf{R}^{n}$ is called countably $(\phi, m)$ rectifiable of class $(k, \alpha)$ if and only if there exist countably many $m$ dimensional submanifolds $M_{j}$ of class $(k, \alpha)$ such that

$$
\phi\left(A \sim \bigcup_{j=1}^{\infty} M_{j}\right)=0 .
$$

A subset $A \subseteq \mathbf{R}^{n}$ is called $(\phi, m)$ rectifiable of class $(k, \alpha)$ if it is countably $(\phi, m)$ rectifiable of class $(k, \alpha)$ and $\phi(A)<\infty$.

Some further notation. Suppose $1 \leq m \leq n$ and $k \geq 1$ are integers, $0 \leq \alpha \leq 1$, $a \in \mathbf{R}^{n}, T \in \mathbf{G}(n, m), 0 \leq \kappa<\infty$ and suppose $f: T \rightarrow T^{\perp}$ is a function such that $f\left(T_{\natural}(a)\right)=T_{\natural}^{\perp}(a)$.

Then we define 9

$$
\mathbf{X}_{k, \alpha}(a, T, f, \kappa)=\mathbf{R}^{n} \cap\left\{z:\left|f\left(T_{\natural}(z)\right)-T_{\natural}^{\perp}(z)\right| \leq \kappa\left|T_{\natural}(z-a)\right|^{k+\alpha}\right\} ;
$$

alternatively $\mathbf{X}_{k}(a, T, f, \kappa)=\mathbf{X}_{k, 0}(a, T, f, \kappa)$. If $f(\chi)=T_{\natural}^{\perp}(a)$ for every $\chi \in T$ then we abbreviate $\mathbf{X}(a, T, \kappa)=\mathbf{X}_{1}(a, T, f, \kappa)$.

If $0<s<\infty$ and $0<t<\infty$ we define

$$
\mathbf{C}(T, a, s, t)=\mathbf{R}^{n} \cap\left\{x:\left|T_{\natural}(x-a)\right|<s,\left|T_{\natural}^{\perp}(x-a)\right|<t\right\} .
$$

Finally let $\operatorname{gr}(f)=\{\chi+f(\chi): \chi \in T\}$.

Organization of the paper. In section 2 we recall the theory of approximate differentiability for functions because both we use it in the following sections and it provides a scheme for the theory of approximate differentiability for sets we develop later. In section 3 the key concepts of lower and upper approximate tangent cones (see 3.1), approximate differentiability (see 3.8) and approximate differentials (see 3.20) are introduced together with proofs of a basic characterization in 3.14, one part of 1.2 in 3.23 and some illustrative examples in 3.4 and 3.17 At the end of section 3 we generalize to rectifiable sets of class 2 the classical equation relating the differential of a normal vector field and the second fundamental form of a submanifold of class 2. In section 4, after giving in 4.8 an equivalent formulation of the notion of pointwise differentiability of order 1 for sets, we prove a result enlightening the relation between approximate differentiability and pointwise differentiability of higher order for sets in 4.11 and a basic estimate for the approximate second fundamental form in 4.12 Finally in section 5 we prove that the approximate differentials are Borel maps and the remaining part of 1.2

The content of this paper was part of author's PHD thesis, supervised by Ulrich Menne, submitted at the University of Potsdam.

Acknowledgements. The author is grateful to Ulrich Menne, who suggested this problem, carefully read the original manuscript and provided the author with a very detailed list of comments, corrections and improvements.

This work was developed while the author was financially supported by the "IMPRS for Geometric Analysis, Gravitation and String Theory" and the "IMPRS for Mathematical and Physical Aspects for Gravitation, Cosmology and Quantum Field Theory".

\footnotetext{
${ }^{8}$ When $\phi=\mathscr{H}^{m}$ this notion has been introduced in AS94, 3.1].

${ }^{9}$ Compare this definition with similar ones introduced in [Fed69] 3.3.1] and [Mat95, 15.12].
} 


\section{Approximate differentiability for functions}

2.1 Definition. Let $f$ be a function mapping a subset of $\mathbf{R}^{m}$ into some set $Y$ and let $a \in \mathbf{R}^{m}$. If $Y$ is a normed vector space, a point $y \in Y$ is the approximate limit of $f$ at $a$ if and only if

$$
\boldsymbol{\Theta}^{m}\left(\mathscr{L}^{m}\left\llcorner\mathbf{R}^{m} \sim\{x:|f(x)-y| \leq \epsilon\}, a\right)=0 \quad \text { for every } \epsilon>0\right.
$$

and we denote it by ap $\lim _{x \rightarrow a} f(x)$. If $Y=\overline{\mathbf{R}}$, a point $t \in \overline{\mathbf{R}}$ is the approximate upper limit of $f$ at $a$ if and only if

$$
t=\inf \left\{s: \Theta^{m}\left(\mathscr{L}^{m}\llcorner\{x: f(x)>s\}, a)=0\right\}\right.
$$

and we denote it by ap $\lim \sup _{x \rightarrow a} f(x)$.

2.2 Remark. This concept is a special case of [Fed69, 2.9.12].

2.3 Definition. Let $1 \leq m<n$ and $k \geq 0$ be integers, $0 \leq \alpha \leq 1, A \subset \mathbf{R}^{m}$, $f: A \rightarrow \mathbf{R}^{n-m}$ and $a \in \mathbf{R}^{m}$.

We say that $f$ is approximately differentiable of order $(k, \alpha)$ at a $(f$ is approximately differentiable of order $k$ at $a$ if $\alpha=0$ ) if

$$
\boldsymbol{\Theta}^{m}\left(\mathscr{L}^{m}\left\llcorner\mathbf{R}^{m} \sim A, a\right)=0\right.
$$

and there exists a polynomial function $P: \mathbf{R}^{m} \rightarrow \mathbf{R}^{n-m}$ of degree at most $k$ such that $P(a)=f(a)$ if $a \in A$,

$$
\operatorname{ap} \lim _{x \rightarrow a} \frac{|f(x)-P(x)|}{|x-a|^{k}}=0 \text { if } \alpha=0, \quad \operatorname{ap} \limsup _{x \rightarrow a} \frac{|f(x)-P(x)|}{|x-a|^{k+\alpha}}<\infty \text { if } \alpha>0 .
$$

2.4 Remark. The condition $\boldsymbol{\Theta}^{m}\left(\mathscr{L}^{m}\left\llcorner\mathbf{R}^{m} \sim A, a\right)=0\right.$ in 2.3 is redundant if $\alpha=0$. Moreover, employing a classical result due to De Giorgi, see Cam64, Lemma 2.I], we deduce that the polynomial function $P$ in 2.3 is uniquely determined by $f$ and $a$.

2.5 Definition. Let $A \subset \mathbf{R}^{m}$ and let $f: A \rightarrow \mathbf{R}^{n-m}$. For every non negative integer $k$ the function ap $\mathrm{D}^{k} f$ is defined to be the function whose domain consists of all $a \in \mathbf{R}^{m}$ such that $f$ is approximately differentiable of order $k$ at $a$ and whose value at $a$ equals $\mathrm{D}^{k} P(a)$, where $P$ satisfies 2.3 .

2.6 Remark. If $a \in A \subset \mathbf{R}^{m}$ and $f: A \rightarrow \mathbf{R}^{n-m}$ then $f$ is approximately differentiable of order 0 at $a$ if and only if $f$ is $\left(\mathscr{L}^{m}, V\right)$ approximately continuous 10 at $a$. In this case ap $\mathrm{D}^{0} f(a)=f(a)$. Here $V$ is the standard $\mathscr{L}^{m}$ Vitali relation, $V=\left\{(a, \mathbf{B}(a, r)): a \in \mathbf{R}^{m}, 0<r<\infty\right\}$.

In case $a \in A$ the notion of approximate differentiability of order 1 has been introduced in Fed69, 3.1.2].

2.7 Lemma. Suppose $1 \leq m<n$ are integers, $A \subseteq \mathbf{R}^{m}, a \in \mathbf{R}^{m}, f: A \rightarrow$ $\mathbf{R}^{n-m}, \gamma \geq 1,0<M<\infty$ and $0 \leq \lambda<\infty$ such that

$$
\limsup _{r \rightarrow 0+} \frac{\mathscr{L}^{m}\left(\mathbf{B}(a, r) \cap\left\{x:|f(x)|>\lambda r^{\gamma}\right\}\right)}{\boldsymbol{\alpha}(m) r^{m}}<M .
$$

Then $\boldsymbol{\Theta}^{* m}\left(\mathscr{L}^{m}\left\llcorner\left\{x:|f(x)|>2^{\gamma} \lambda|x-a|^{\gamma}\right\}, a\right)<M\left(1-2^{-m}\right)^{-1}\right.$.

${ }^{10}$ See [Fed69, 2.9.12]. 
Proof. Let $\delta>0$ such that

$$
\mathscr{L}^{m}\left(\mathbf{B}(a, r) \cap\left\{x:|f(x)|>\lambda r^{\gamma}\right\}\right)<M \boldsymbol{\alpha}(m) r^{m} \quad \text { for } 0<r \leq \delta .
$$

Therefore for $0<r \leq \delta$ we observe

$$
\begin{aligned}
& \mathbf{B}(a, r) \cap\left\{x:|f(x)|>2^{\gamma} \lambda|x-a|^{\gamma}\right\} \\
& \quad=\{a\} \cup \bigcup_{i=0}^{\infty}\left(\mathbf{B}\left(a, r / 2^{i}\right) \sim \mathbf{B}\left(a, r / 2^{i+1}\right)\right) \cap\left\{x:|f(x)|>2^{\gamma} \lambda|x-a|^{\gamma}\right\} \\
& \quad \subseteq\{a\} \cup \bigcup_{i=0}^{\infty} \mathbf{B}\left(a, r / 2^{i}\right) \cap\left\{x:|f(x)|>\lambda\left(r / 2^{i}\right)^{\gamma}\right\}, \\
& \mathscr{L}^{m}\left(\mathbf{B}(a, r) \cap\left\{x:|f(x)|>2^{\gamma} \lambda|x-a|^{\gamma}\right\}\right)<M \boldsymbol{\alpha}(m) r^{m}\left(1-2^{-m}\right)^{-1}
\end{aligned}
$$

and the conclusion follows.

2.8 Theorem. Let $1 \leq m<n$ and $k \geq 1$ be integers, $0 \leq \alpha \leq 1, A \subset \mathbf{R}^{m}$, $a \in \mathbf{R}^{m}$ and $f: A \rightarrow \mathbf{R}^{n-m}$.

Then $f$ is approximately differentiable of order $(k, \alpha)$ at a if and only if there exists a function $g: \mathbf{R}^{m} \rightarrow \mathbf{R}^{n-m}$ pointwise differentiable of order $(k, \alpha)$ at a such that $f(a)=g(a)$ if $a \in A$ and

$$
\boldsymbol{\Theta}^{m}\left(\mathscr{L}^{m}\left\llcorner\mathbf{R}^{m} \sim\{x: g(x)=f(x)\}, a\right)=0 .\right.
$$

In this case $\operatorname{ptD}^{i} g(a)=\operatorname{apD}^{i} f(a)$ for $i=0, \ldots, k$.

Proof. Suppose $f$ is approximately differentiable of order $(k, \alpha)$ at $a$ and $\alpha=0$. There exists a polynomial function $P: \mathbf{R}^{m} \rightarrow \mathbf{R}^{n-m}$ of degree at most $k$ such that, if for every integer $i \geq 1$ we define $S_{i}=\left\{x:|f(x)-P(x)|<i^{-1}|x-a|^{k}\right\}$, then there exists $\delta_{i}>0$ such that $\mathscr{L}^{m}\left(\mathbf{B}(a, r) \sim S_{i}\right)<2^{-i} r^{m}$ for $0<r \leq \delta_{i}$. We can assume $\delta_{i+1}<\delta_{i}$ for each $i \geq 1$ and $\delta_{i} \rightarrow 0$ as $i \rightarrow \infty$. Let

$$
T=\bigcup_{i=1}^{\infty}\left[S_{i} \cap \mathbf{B}\left(a, \delta_{i}\right) \sim \mathbf{B}\left(a, \delta_{i+1}\right)\right] .
$$

If $r>0$ and $j \geq 1$ is an integer such that $\delta_{j+1}<r \leq \delta_{j}$ we compute

$$
\mathscr{L}^{m}(\mathbf{B}(a, r) \sim T) \leq \mathscr{L}^{m}\left(\mathbf{B}(a, r) \sim S_{j}\right)+\sum_{l=j+1}^{\infty} \mathscr{L}^{m}\left(\mathbf{B}\left(a, \delta_{l}\right) \sim S_{l}\right)<r^{m} \sum_{l=j}^{\infty} 2^{-l}
$$

and we conclude $\boldsymbol{\Theta}^{m}\left(\mathscr{L}^{m}\left\llcorner\mathbf{R}^{m} \sim T, a\right)=0\right.$. Moreover

$$
\lim _{T \ni x \rightarrow a} \frac{|f(x)-P(x)|}{|x-a|^{k}}=0 .
$$

If we define $g: \mathbf{R}^{m} \rightarrow \mathbf{R}^{n-m}$ as $g(x)=f(x)$ if $x \in T$ and $g(x)=P(x)$ if $x \in \mathbf{R}^{m} \sim T$, then we have $\boldsymbol{\Theta}^{m}\left(\mathscr{L}^{m}\left\llcorner\mathbf{R}^{m} \sim\{x: g(x)=f(x)\}, a\right)=0\right.$,

$$
\lim _{x \rightarrow a} \frac{|g(x)-P(x)|}{|x-a|^{k}}=0 \text { and } \quad g(a)=P(a),
$$

since $a \notin T$. If $\alpha>0$, once we have chosen $0 \leq \lambda<\infty$ such that

$$
\operatorname{ap} \limsup _{x \rightarrow a} \frac{|f(x)-P(x)|}{|x-a|^{k+\alpha}}<\lambda,
$$

we can use the same argument above replacing the sets $S_{i}$ with the set

$$
S=\left\{x:|f(x)-P(x)|<\lambda|x-a|^{k+\alpha}\right\} .
$$


2.9 Remark. The proof of 2.8 has been adapted from Fed69, 3.2.16] and Fed69, 3.1.22].

2.10 Remark. Let $1 \leq m<n$ and $k \geq 1$ be integers, $0 \leq \alpha \leq 1$, let $A \subset \mathbf{R}^{m}$ be $\mathscr{L}^{m}$ measurable and let $f: A \rightarrow \mathbf{R}^{n-m}$ be $\mathscr{L}^{m}\llcorner A$ measurable. If there exist countably many functions $g_{j}: \mathbf{R}^{m} \rightarrow \mathbf{R}^{n-m}$ of class $(k, \alpha)$ such that

$$
\mathscr{L}^{m}\left(A \sim \bigcup_{j=1}^{\infty} A_{j}\right)=0
$$

where $A_{j}=A \cap\left\{z: g_{j}(z)=f(z)\right\}$ for $j \geq 1$, then using [Fed69, 2.10.19(4)] and 2.8 we can easily prove that $f$ is approximately differentiable of order $(k, \alpha)$ at $\mathscr{L}^{m}$ a.e. $a \in A$ and, for each $j \geq 1$,

$$
\mathrm{D}^{i} g_{j}(z)=\operatorname{apD}^{i} f(z) \text { for } \mathscr{L}^{m} \text { a.e. } z \in A_{j} \text { and } i=0, \ldots, k \text {. }
$$

2.11 Theorem. Let $1 \leq m<n$ and $k \geq 0$ be integers, $A \subset \mathbf{R}^{m}$ and let $f: A \rightarrow \mathbf{R}^{n-m}$ be approximately differentiable of order $(k, 1)$ at $\mathscr{L}^{m}$ a.e. $a \in A$. Then the following statements hold.

(1) $f$ is approximately differentiable of order $k+1$ at $\mathscr{L}^{m}$ a.e. $x \in A$.

(2) $A$ is $\mathscr{L}^{m}$ measurable and the functions ap $\mathrm{D}^{i} f$ are $\mathscr{L}^{m}\llcorner A$ measurable for $i=0, \ldots, k+1$.

(3) There exist countably many functions $g_{j}: \mathbf{R}^{m} \rightarrow \mathbf{R}^{n-m}$ of class $k+1$ such that

$$
\mathscr{L}^{m}\left(A \sim \bigcup_{j=1}^{\infty}\left\{x: g_{j}(x)=f(x)\right\}\right)=0 .
$$

Proof. First we observe that $A$ is $\mathscr{L}^{m}$ measurable, $f$ is $\left(\mathscr{L}^{m}, V\right)$ approximately continuou $\sqrt{11}$ at $\mathscr{L}^{m}$ a.e. $a \in A$ and $f$ is $\mathscr{L}^{m}\llcorner A$ measurable by [Fed69, 2.9.11, 2.9.13].

If $k=0$ the conclusions are consequences of [Fed69, 3.1.8, 3.1.4, 3.1.16] respectively. We use induction over $k$. Since $f$ is approximately differentiable of order $(k-1,1)$ at $\mathscr{L}^{m}$ a.e. point of $A$ we inductively assume that ap $\mathrm{D}^{i} f$ are $\mathscr{L}^{m}\llcorner A$ measurable for $i=0, \ldots, k$. We use now [sa87, Theorem 2] and [Fed69, $3.1 .15]$ to deduce the existence of countably many functions $g_{j}: \mathbf{R}^{m} \rightarrow \mathbf{R}^{n}$ of class $k+1$ satisfying (3). Now (11) and (2) follow from 2.10.

2.12 Theorem. Suppose $1 \leq m<n$ and $k \geq 1$ are integers, $0 \leq \alpha \leq 1$, $A \subset \mathbf{R}^{m}$ and $f: A \rightarrow \mathbf{R}^{n-m}$ is approximately differentiable of order $(k, \alpha)$ at $\mathscr{L}^{m}$ a.e. $a \in A$.

Then the following statements hold.

(1) $A$ is $\mathscr{L}^{m}$ measurable and the functions ap $\mathrm{D}^{i} f$ are $\mathscr{L}^{m}\llcorner A$ measurable for $i=0, \ldots, k$.

(2) There exist countably many functions $g_{j}: \mathbf{R}^{m} \rightarrow \mathbf{R}^{n-m}$ of class $(k, \alpha)$ such that

$$
\mathscr{L}^{m}\left(A \sim \bigcup_{j=1}^{\infty}\left\{x: g_{j}(x)=f(x)\right\}\right)=0 .
$$

Proof. Since $f$ is approximately differentiable of order $(k-1,1)$ at every $x \in A$ then (11) follows from 2.11(2). Now we can apply [sa87, Theorem 1] if $\alpha=0$ or [Isa87, Theorem 2] if $\alpha>0$ to get (2).

\footnotetext{
${ }^{11}$ As usual, $V=\left\{(a, \mathbf{B}(a, r)): a \in \mathbf{R}^{m}, 0<r<\infty\right\}$.
} 


\section{Approximate differentiability for sets}

3.1 Definition. Suppose $X$ is a normed vector space, $\phi$ is a measure over $X$, $m$ is a positive integer and $a \in X$.

We define the $m$ dimensional approximate upper tangent cone of $\phi$ at a by 12

$$
\operatorname{Tan}^{* m}(\phi, a)=X \cap\left\{v: \boldsymbol{\Theta}^{* m}(\phi\llcorner\mathbf{E}(a, v, \epsilon), a)>0 \text { for every } \epsilon>0\}\right.
$$

and the $m$ dimensional approximate lower tangent cone of $\phi$ at $a$ as the set $\operatorname{Tan}_{*}^{m}(\phi, a)$ of $v \in X$ such that for every $\epsilon>0$ there exists $\eta>0$ such that

$$
\phi(\mathbf{U}(a+r v, \epsilon r)) \geq \eta r^{m} \quad \text { whenever } 0<r \leq \eta .
$$

In case $\operatorname{Tan}^{* m}(\phi, a)=\operatorname{Tan}_{*}^{m}(\phi, a)$, this set is denoted by $\operatorname{Tan}^{m}(\phi, a)$ and we call it the $m$ dimensional approximate tangent cone of $\phi$ at a.

3.2 Remark. Evidently $\operatorname{Tan}_{*}^{m}(\phi, a) \subseteq \operatorname{Tan}^{* m}(\phi, a)$. Moreover one may easily verify that $\operatorname{Tan}_{*}^{m}(\phi, a)$ and $\operatorname{Tan}^{* m}(\phi, a)$ are closed cones. Finally

$$
\Theta^{* m}(\phi, a)>0 \quad\left[\Theta_{*}^{m}(\phi, a)>0\right] \Longleftrightarrow 0 \in \operatorname{Tan}^{* m}(\phi, a) \quad\left[0 \in \operatorname{Tan}_{*}^{m}(\phi, a)\right] .
$$

3.3 Remark. Observe that, in this case, our notation does not agree with Fed69, 3.2.16]. In fact, $\operatorname{Tan}^{* m}(\phi, a)$ is denoted by $\operatorname{Tan}^{m}(\phi, a)$ in [Fed69, 3.2.16].

It is often useful to recall that if $C$ is a compact subset of $X \sim \operatorname{Tan}^{* m}(\phi, a)$ and $T=\{a+r v: r \geq 0, v \in C\}$ then $\boldsymbol{\Theta}^{m}(\phi\llcorner T, a)=0$. This is proved in Fed69, 3.2.16].

3.4 Remark. It is natural to consider the following cone

$$
T=X \cap\left\{v: \boldsymbol{\Theta}_{*}^{m}(\phi\llcorner\mathbf{E}(a, v, \epsilon), a)>0 \text { for every } \epsilon>0\} .\right.
$$

Evidently $\operatorname{Tan}_{*}^{m}(\phi, a) \subseteq T$, but simple examples show that the opposite inclusion does not hold. In fact, if we consider $X=\mathbf{R}, \phi=\mathscr{L}^{1}\llcorner A, m=1$ and $a=0$, where

$$
A=\bigcup_{i=0}^{\infty} \mathbf{R} \cap\left\{t: 2^{-2 i-1}<|t|<2^{-2 i}\right\},
$$

then $\operatorname{Tan}_{*}^{1}(\phi, 0)=\{0\}$ and $T=\mathbf{R}$.

3.5 Remark. Suppose $1 \leq m \leq n$ are integers, $A \subseteq \mathbf{R}^{n}, B \subseteq \mathbf{R}^{n}$ and $a \in \mathbf{R}^{n}$.

If $\boldsymbol{\Theta}^{m}\left(\mathscr{H}^{m}\llcorner A \sim B, a)=0\right.$ then it is not difficult to see that

$$
\begin{aligned}
\operatorname{Tan}_{*}^{m}\left(\mathscr{H}^{m}\llcorner A, a)\right. & \subseteq \operatorname{Tan}_{*}^{m}\left(\mathscr{H}^{m}\llcorner B, a),\right. \\
\operatorname{Tan}^{* m}\left(\mathscr{H}^{m}\llcorner A, a)\right. & \subseteq \operatorname{Tan}^{* m}\left(\mathscr{H}^{m}\llcorner B, a) .\right.
\end{aligned}
$$

3.6 Lemma. Suppose $1 \leq m \leq n$ are integers, $A \subseteq \mathbf{R}^{n}, a \in \mathbf{R}^{n}$ and $T \in \mathbf{G}(n, m)$.

Then the following three conditions are equivalent:

(1) $\operatorname{Tan}^{* m}\left(\mathscr{H}^{m}\llcorner A, a) \subseteq T\right.$,

(2) $\boldsymbol{\Theta}^{m}\left(\mathscr{H}^{m}\llcorner A \sim \mathbf{X}(a, T, \epsilon), a)=0\right.$ whenever $\epsilon>0$,

\footnotetext{
${ }^{12}$ As in [Fed69] 3.2.16], $\mathbf{E}(a, v, \epsilon)=X \cap\{x:|r(x-a)-v|<\epsilon$ for some $0<r<\infty\}$.
} 
(3) whenever $\epsilon>0$

$$
\lim _{r \rightarrow 0} \frac{\mathscr{H}^{m}\left(A \cap \mathbf{B}(a, r) \cap\left\{z:\left|T_{\natural}^{\perp}(z-a)\right|>\epsilon r\right\}\right)}{\boldsymbol{\alpha}(m) r^{m}}=0 .
$$

Proof. The fact that (11) implies (2) is a consequence of 3.3 and the fact that (3) follows from (2) is evident. If the condition in (3) holds for some $\epsilon>0$ then we can argue as in 2.7 to show that

$$
\lim _{r \rightarrow 0} \frac{\mathscr{H}^{m}\left(A \cap \mathbf{B}(a, r) \cap\left\{z:\left|T_{\natural}^{\perp}(z-a)\right|>2 \epsilon|z-a|\right\}\right)}{\boldsymbol{\alpha}(m) r^{m}}=0 .
$$

Therefore (3) implies (11).

3.7 Lemma. Let $1 \leq m \leq n$ and $k \geq 1$ be integers, $0 \leq \alpha \leq 1,0 \leq \lambda<\infty$, $0<M<\infty, A \subseteq \mathbf{R}^{n}, a \in \mathbf{R}^{n}, T \in \mathbf{G}(n, m)$ and let $f: T \rightarrow T^{\perp}$ be a function of class 1 such that $f\left(T_{\natural}(a)\right)=T_{\natural}^{\perp}(a)$ and $\mathrm{D} f\left(T_{\mathfrak{\natural}}(a)\right)=0$. Suppose

$$
\begin{gathered}
\lim _{r \rightarrow 0} \frac{\mathscr{H}^{m}\left(A \cap \mathbf{B}(a, r) \cap\left\{z:\left|T_{\natural}^{\perp}(z)-f\left(T_{\natural}(z)\right)\right|>\epsilon r\right\}\right)}{\boldsymbol{\alpha}(m) r^{m}}=0 \text { for every } \epsilon>0, \\
\limsup _{r \rightarrow 0} \frac{\mathscr{H}^{m}\left(A \cap \mathbf{B}(a, r) \cap\left\{z:\left|T_{\natural}^{\perp}(z)-f\left(T_{\natural}(z)\right)\right|>\lambda r^{k+\alpha}\right\}\right)}{\boldsymbol{\alpha}(m) r^{m}}<M .
\end{gathered}
$$

Then

$$
\Theta^{* m}\left(\mathscr{H}^{m}\left\llcorner A \sim \mathbf{X}_{k, \alpha}(a, T, f, \kappa), a\right)<M\left(1-2^{-m}\right)^{-1}\right.
$$

for every $\kappa>2^{k+\alpha} \lambda$.

Proof. Arguing as in the proof of 2.7 we conclude that

$\Theta^{* m}\left(\mathscr{H}^{m}\left\llcorner A \cap\left\{z:\left|f\left(T_{\natural}(z)\right)-T_{\natural}^{\perp}(z)\right|>2^{k+\alpha} \lambda|z-a|^{k+\alpha}\right\}, a\right)<M\left(1-2^{-m}\right)^{-1}\right.$.

Since $\operatorname{D} f\left(T_{\natural}(a)\right)=0$ we can easily get that

$$
\lim _{r \rightarrow 0} \frac{\mathscr{H}^{m}\left(A \cap \mathbf{B}(a, r) \cap\left\{z:\left|T_{\natural}^{\perp}(z-a)\right|>\epsilon r\right\}\right)}{\boldsymbol{\alpha}(m) r^{m}}=0 \text { for every } \epsilon>0
$$

and applying 3.6 we conclude that $\boldsymbol{\Theta}^{m}\left(\mathscr{H}^{m}\llcorner A \sim \mathbf{X}(a, T, \epsilon), a)=0\right.$. Since

$$
\begin{aligned}
& \mathbf{X}(a, T, \epsilon) \cap\left\{z:\left|f\left(T_{\natural}(z)\right)-T_{\natural}^{\perp}(z)\right| \leq 2^{k+\alpha} \lambda|z-a|^{k+\alpha}\right\} \\
& \quad \subseteq \mathbf{X}_{k, \alpha}\left(a, T, f, 2^{k+\alpha} \lambda\left(1+\epsilon^{2}\right)^{(k+\alpha) / 2}\right) \quad \text { for every } \epsilon>0,
\end{aligned}
$$

the conclusion follows.

3.8 Definition. Let $n \geq 1$ and $k \geq 1$ be integers, $0 \leq \alpha \leq 1, A \subseteq \mathbf{R}^{n}, a \in \mathbf{R}^{n}$ and $A_{1}=\{x-a: x \in A\}$. We say that $A$ is approximately differentiable of order $(k, \alpha)$ at $a$ if there exist an integer $1 \leq m \leq n, T \in \mathbf{G}(n, m)$ and a polynomial function $P: T \rightarrow T^{\perp}$ of degree at most $k$ such that $P(0)=0, \mathrm{D} P(0)=0$ and the following two conditions hold.

(1) For every $\epsilon>0$ there exists $\rho>0$ and $\eta>0$ such that

$$
\mathscr{H}^{m}\left(\mathbf{C}(T, z, \epsilon r, \epsilon r) \cap A_{1}\right) \geq \eta r^{m}
$$

for every $z \in T \cap \mathbf{B}(0, r)$ and $0 \leq r \leq \rho$. 
(2) For every $\epsilon>0$

$$
\lim _{r \rightarrow 0} \frac{\mathscr{H}^{m}\left(A_{1} \cap \mathbf{B}(0, r) \cap\left\{z: \boldsymbol{\delta}_{\operatorname{gr}(P)}(z)>\epsilon r^{k}\right\}\right)}{\boldsymbol{\alpha}(m) r^{m}}=0
$$

and, if $\alpha>0$, there exists $0 \leq \lambda<\infty$ such that

$$
\lim _{r \rightarrow 0} \frac{\mathscr{H}^{m}\left(A_{1} \cap \mathbf{B}(0, r) \cap\left\{z: \boldsymbol{\delta}_{\operatorname{gr}(P)}(z)>\lambda r^{k+\alpha}\right\}\right)}{\boldsymbol{\alpha}(m) r^{m}}=0 .
$$

3.9 Remark. If $k=1$ and $\alpha=0$ the conditions in 3.8 are equivalent to Mat95. 15.7].

3.10 Remark. We prove that the condition

$$
T \subseteq \operatorname{Tan}_{*}^{m}\left(\mathscr{H}^{m}\llcorner A, a)\right.
$$

is necessary and sufficient to have 3.8(1). The condition is clearly necessary. To prove the sufficiency assume $a=0$, suppose $0<\epsilon<1$ and observe there exist an integer $l \geq 1, v_{1}, \ldots, v_{l} \in \mathbf{S}^{n-1} \cap T$ and a positive number $\eta$ such that

$$
\begin{gathered}
T \cap \mathbf{S}^{n-1} \subseteq \bigcup_{i=1}^{l} \mathbf{U}\left(v_{i}, \epsilon\right) \cap T, \\
\mathscr{H}^{m}\left(A \cap \mathbf{U}\left(r v_{i}, \epsilon r\right)\right) \geq \eta \epsilon^{-m} r^{m} \quad \text { whenever } 0<r \leq \eta \text { and } i=1, \ldots, l .
\end{gathered}
$$

Since $\boldsymbol{\Theta}_{*}^{m}\left(\mathscr{H}^{m}\llcorner A, 0)>0\right.$ by 3.2 we can choose $\eta>0$ smaller, if necessary, in order to have

$$
\mathscr{H}^{m}(A \cap \mathbf{U}(0, \epsilon r)) \geq \eta r^{m} \quad \text { whenever } 0<r \leq \eta .
$$

We fix $0<r \leq \eta$ and $z \in \mathbf{B}(0, r)$. If $|z| \leq \epsilon r$ then $\mathbf{U}(0, \epsilon r) \subseteq \mathbf{U}(z, 2 \epsilon r)$ and

$$
\mathscr{H}^{m}(A \cap \mathbf{U}(z, 2 \epsilon r)) \geq \eta r^{m} .
$$

If $|z| \geq \epsilon r$ then we choose $1 \leq i \leq l$ such that $\left|(z /|z|)-v_{i}\right|<\epsilon$ and we observe

$$
\begin{aligned}
& \mathbf{U}\left(|z| v_{i},|z| \epsilon\right) \subseteq \mathbf{U}(z, 2 \epsilon|z|) \subseteq \mathbf{U}(z, 2 \epsilon r), \\
& \mathscr{H}^{m}(A \cap \mathbf{U}(z, 2 \epsilon r)) \geq \eta \epsilon^{-m}|z|^{m} \geq \eta r^{m} .
\end{aligned}
$$

3.11 Lemma. Suppose $1 \leq m \leq n$ are integers, $0<r<\infty, w \in \mathbf{R}^{n} \cap \mathbf{B}(0, r)$, $T \in \mathbf{G}(n, m)$ and $f: T \rightarrow T^{\perp}$ is a locally Lipschitzian function such that $f(0)=0$. .

Then $\boldsymbol{\delta}_{\operatorname{gr} f}(w) \leq\left|T_{\natural}^{\perp}(w)-f\left(T_{\natural}(w)\right)\right| \leq(2+\operatorname{Lip}(f \mid \mathbf{B}(0,2 r))) \boldsymbol{\delta}_{\operatorname{gr} f}(w)$.

Proof. If we choose $\chi \in T$ so that $\boldsymbol{\delta}_{\operatorname{gr} f}(w)=|w-\chi-f(\chi)|$ then $\chi \in \mathbf{B}(0,2 r)$ and we get

$$
\begin{aligned}
\boldsymbol{\delta}_{\operatorname{gr} f}(w) & \leq\left|T_{\natural}^{\perp}(w)-f\left(T_{\natural}(w)\right)\right| \\
& \leq|w-\chi-f(\chi)|+\left|\chi+f(\chi)-T_{\natural}(w)-f\left(T_{\natural}(w)\right)\right| \\
& \leq(2+\operatorname{Lip}(f \mid \mathbf{B}(0,2 r))) \boldsymbol{\delta}_{\operatorname{gr} f}(w) .
\end{aligned}
$$

3.12 Lemma. Suppose $1 \leq m \leq n$ are integers, $\gamma>0, A \subseteq \mathbf{R}^{n}, B \subseteq \mathbf{R}^{n}$ such that $0 \in \operatorname{Clos} B, f: \mathbf{R}^{n} \rightarrow \mathbf{R}^{n}$ is an univalent map onto $\mathbf{R}^{n}$ such that $f(0)=0$ and $f$ and $f^{-1}$ are locally Lipschitzian maps.

Then the following two conditions are equivalent. 
(1) For every $\epsilon>0$ [for some $0 \leq \epsilon<\infty$ ]

$$
\lim _{r \rightarrow 0} \frac{\mathscr{H}^{m}\left(A \cap \mathbf{B}(0, r) \cap\left\{z: \boldsymbol{\delta}_{B}(z)>\epsilon r^{\gamma}\right\}\right)}{\boldsymbol{\alpha}(m) r^{m}}=0 .
$$

(2) For every $\epsilon>0$ [for some $0 \leq \epsilon<\infty$ ]

$$
\lim _{r \rightarrow 0} \frac{\mathscr{H}^{m}\left(f[A] \cap \mathbf{B}(0, r) \cap\left\{z: \boldsymbol{\delta}_{f[B]}(z)>\epsilon r^{\gamma}\right\}\right)}{\boldsymbol{\alpha}(m) r^{m}}=0 .
$$

Proof. Since $f[\operatorname{Clos} B]=\operatorname{Clos} f[B]$ we assume $B$ to be closed. Moreover if we prove one implication we immediately get the other one. Therefore we prove that (11) implies (2). Suppose $1<\Gamma<\infty$ is such that

$$
\begin{gathered}
\Gamma^{-1}|z-w| \leq|f(z)-f(w)| \leq \Gamma|z-w| \\
\Gamma^{-1}|z-w| \leq\left|f^{-1}(z)-f^{-1}(w)\right| \leq \Gamma|z-w|
\end{gathered}
$$

whenever $z, w \in \mathbf{B}(0,2)$. Evidently it is enough to show that

$$
\begin{gathered}
f^{-1}\left[f[A] \cap \mathbf{B}\left(0, r / \Gamma^{2}\right) \cap\left\{w: \boldsymbol{\delta}_{f[B]}(w)>\epsilon r^{\gamma}\right\}\right] \\
\subseteq A \cap \mathbf{B}(0, r / \Gamma) \cap\left\{w: \boldsymbol{\delta}_{B}(w)>\Gamma^{-1} \epsilon r^{\gamma}\right\}
\end{gathered}
$$

for $\epsilon>0$ and $0<r \leq 1$. Suppose $z \in f[A] \cap \mathbf{B}\left(0, r / \Gamma^{2}\right)$ such that $\boldsymbol{\delta}_{f[B]}(z)>\epsilon r^{\gamma}$. Let $w \in f[B]$ such that $\left|f^{-1}(z)-f^{-1}(w)\right|=\boldsymbol{\delta}_{B}\left(f^{-1}(z)\right)$ and observe

$$
\begin{gathered}
\boldsymbol{\delta}_{B}\left(f^{-1}(z)\right) \leq\left|f^{-1}(z)\right|, \quad\left|f^{-1}(w)\right| \leq 2\left|f^{-1}(z)\right| \leq 2 \Gamma|z| \leq 2 \Gamma^{-1} r \leq 2, \\
|w| \leq \Gamma\left|f^{-1}(w)\right| \leq 2 r \leq 2, \\
\boldsymbol{\delta}_{B}\left(f^{-1}(z)\right) \geq \Gamma^{-1}|z-w| \geq \Gamma^{-1} \boldsymbol{\delta}_{f[B]}(z)>\Gamma^{-1} \epsilon r^{\gamma} .
\end{gathered}
$$

3.13 Lemma. Let $1 \leq m \leq n$ and $k \geq 1$ be integers, $T \in \mathbf{G}(n, m)$ and let $P: T \rightarrow T^{\perp}$ and $Q: T \rightarrow T^{\perp}$ be polynomial functions of degree at most $k$ such that $P(0)=0$ and $\mathrm{D}^{i} Q(0)=0$ for $i=0, \ldots, k-1$. Suppose for every $\epsilon>0$ there exists $\rho>0$ such that

$$
\mathbf{C}\left(T, z, \epsilon r, \epsilon r^{k}\right) \cap\left\{w: \boldsymbol{\delta}_{\operatorname{gr}(Q)}(w) \leq \epsilon r^{k}\right\} \neq \varnothing
$$

whenever $z \in \operatorname{gr}(P) \cap \mathbf{B}(0, r)$ and $0<r \leq \rho$.

Then $P=Q$.

Proof. Let $0 \leq c<\infty$ such that $|P(\chi)| \leq c|\chi|$ whenever $\chi \in T \cap \mathbf{B}(0,1)$. If $0<\epsilon \leq 1$ and $0<\rho \leq 1$ are as in the hypothesis, $\chi \in \mathbf{B}\left(0,(1+c)^{-1} \rho\right) \cap T$ and $z=\chi+P(\chi)$ then $|z| \leq(1+c)|\chi| \leq \rho$. Therefore there exists

$$
w \in \mathbf{C}\left(T, z, \epsilon(1+c)|\chi|, \epsilon(1+c)^{k}|\chi|^{k}\right)
$$

such that $\boldsymbol{\delta}_{\operatorname{gr}(Q)}(w) \leq \epsilon(1+c)^{k}|\chi|^{k}$. If $y \in \operatorname{gr}(Q)$ is such that $|w-y|=\boldsymbol{\delta}_{\operatorname{gr}(Q)}(w)$ then

$$
\begin{gathered}
\left|T_{\natural}(y)-\chi\right| \leq\left|T_{\natural}(y-w)\right|+\left|T_{\natural}(w)-\chi\right| \leq 2 \epsilon(1+c)^{k}|\chi|, \\
|P(\chi)-Q(\chi)| \leq\left|T_{\natural}^{\perp}(z)-T_{\natural}^{\perp}(w)\right|+\left|T_{\natural}^{\perp}(w)-T_{\natural}^{\perp}(y)\right|+\left|T_{\natural}^{\perp}(y)-Q(\chi)\right|,
\end{gathered}
$$


and the Taylor's formula (see [Fed69, p. 46]) implies

$$
\begin{aligned}
Q\left(T_{\natural}(y)\right)-Q(\chi)= & \sum_{i=1}^{k}\left\langle\left(T_{\natural}(y)-\chi\right)^{i} / i ! \odot \chi^{k-i} /(k-i) !, \mathrm{D}^{k} Q(0)\right\rangle, \\
& \left|Q\left(T_{\natural}(y)\right)-Q(\chi)\right| \leq c_{1} \epsilon|\chi|^{k},
\end{aligned}
$$

where $c_{1}=\left\|\mathrm{D}^{k} Q(0)\right\| \sum_{i=1}^{k} 2^{i}(1+c)^{k i} /(i !(k-i) !)$. Therefore $|Q(\chi)-P(\chi)| \leq$ $\left(2(1+c)^{k}+c_{1}\right) \epsilon|\chi|^{k}$ and the conclusion follows.

3.14 Theorem. Suppose $1 \leq m \leq n$ and $k \geq 1$ are integers, $0 \leq \alpha \leq 1$, $A \subseteq \mathbf{R}^{n}, a \in \mathbf{R}^{n}, A_{1}=\{x-a: x \in A\}, T \in \mathbf{G}(n, m)$ and $P: T \rightarrow T^{\perp}$ is $a$ polynomial function of degree at most $k$ such that $P(0)=0$, D $P(0)=0$.

Then the following two conditions are equivalent.

(1) $T$ and $P$ satisfy 3.8(1) and 3.8(2).

(2) If $P_{i}(\chi)=\left\langle\chi^{i} / i !, D^{i} P(0)\right\rangle$ for $\chi \in T$ and $i=1, \ldots, k$ and

$$
A_{i}=\left\{x-P_{i-1}\left(T_{\natural}(x)\right): x \in A_{i-1}\right\} \quad \text { for } i=2, \ldots, k,
$$

then the following two conditions hold:

(a) for every $i=1, \ldots, k$ and for every $\epsilon>0$ there exist $\rho>0$ and $\eta>0$ such that

$$
\mathscr{H}^{m}\left(\mathbf{C}\left(T, z, \epsilon r, \epsilon r^{i}\right) \cap A_{i}\right) \geq \eta \boldsymbol{\alpha}(m) r^{m}
$$

for every $z \in \operatorname{gr}\left(P_{i}\right) \cap \mathbf{B}(0, r)$ and $0 \leq r \leq \rho$,

(b) for every $i=1, \ldots, k$ and for every $\epsilon>0$

$$
\lim _{r \rightarrow 0} \frac{\mathscr{H}^{m}\left(A_{i} \cap \mathbf{B}(0, r) \cap\left\{z: \boldsymbol{\delta}_{\operatorname{gr}\left(P_{i}\right)}(z)>\epsilon r^{i}\right\}\right)}{\boldsymbol{\alpha}(m) r^{m}}=0
$$

and, if $\alpha>0$, there exists $0 \leq \lambda<\infty$ such that

$$
\lim _{r \rightarrow 0} \frac{\mathscr{H}^{m}\left(A_{k} \cap \mathbf{B}(0, r) \cap\left\{z: \boldsymbol{\delta}_{\operatorname{gr}\left(P_{k}\right)}(z)>\lambda r^{k+\alpha}\right\}\right)}{\boldsymbol{\alpha}(m) r^{m}}=0 .
$$

In this case $P$ is uniquely determined by $a, A$ and $k$,

$$
\Theta_{*}^{m}\left(\mathscr{H}^{m}\llcorner A, a)>0, \operatorname{Tan}_{*}^{m}\left(\mathscr{H}^{m}\llcorner A, a)=\operatorname{Tan}^{* m}\left(\mathscr{H}^{m}\llcorner A, a)=T\right.\right.\right.
$$

and $A$ is approximately differentiable of order $(l, \beta)$ whenever either $l<k$ and $0 \leq \beta \leq 1$ or $l=k$ and $0 \leq \beta \leq \alpha$.

Proof. Assume $a=0$ and suppose $\sup \left\{1, \sum_{j=1}^{k} 2^{j}\left\|\mathrm{D}^{j} P(0)\right\|\right\}<\Gamma<\infty$.

For $i=1, \ldots, k$ we define $Q_{i}=\sum_{j=1}^{i} P_{j}$ and $f_{i}: \mathbf{R}^{n} \rightarrow \mathbf{R}^{n}$ by

$$
f_{i}(x)=x-Q_{i}\left(T_{\natural}(x)\right)+P_{i}\left(T_{\natural}(x)\right) \quad \text { for } x \in \mathbf{R}^{n} .
$$

We observe that for every $i=1, \ldots, k$ the map $f_{i}$ is a diffeomorphism of class $\infty$ onto $\mathbf{R}^{n}$ and, by induction over $i$, one may easily prove that

$$
f_{i}[A]=A_{i}, \quad f_{i}\left[\operatorname{gr} Q_{i}\right]=\operatorname{gr} P_{i} .
$$


Now, using 3.12, it is easy to see that (2) implies (1). Henceforth, we assume (11). First we prove that

$$
\boldsymbol{\delta}_{\operatorname{gr} P}(z) \geq \boldsymbol{\delta}_{\operatorname{gr} Q_{i}}(z)-\Gamma|z|^{i+1} \quad \text { for every } i=1, \ldots, k-1 \text { and } z \in \mathbf{B}(0,1) .
$$

In fact if $w \in \operatorname{gr}(P)$ such that $|z-w|=\boldsymbol{\delta}_{\operatorname{gr}(P)}(z)$ then

$$
\begin{gathered}
|w| \leq 2|z|, \quad\left|P\left(T_{\natural}(w)\right)-Q_{i}\left(T_{\natural}(w)\right)\right| \leq \Gamma|z|^{i+1}, \\
|z-w| \geq\left|z-T_{\natural}(w)-Q_{i}\left(T_{\natural}(w)\right)\right|-\left|Q_{i}\left(T_{\natural}(w)\right)-P\left(T_{\natural}(w)\right)\right| \\
\geq \boldsymbol{\delta}_{\operatorname{gr}} Q_{i}(z)-\Gamma|z|^{i+1} .
\end{gathered}
$$

It follows, for $i=1, \ldots, k$, that

$$
\lim _{r \rightarrow 0} \frac{\mathscr{H}^{m}\left(A_{1} \cap \mathbf{B}(0, r) \cap\left\{z: \boldsymbol{\delta}_{\operatorname{gr}\left(Q_{i}\right)}(z)>\epsilon r^{i}\right\}\right)}{\boldsymbol{\alpha}(m) r^{m}}=0 \quad \text { for every } \epsilon>0 .
$$

and, for $i=1, \ldots, k-1$ that

$$
\lim _{r \rightarrow 0} \frac{\mathscr{H}^{m}\left(A_{1} \cap \mathbf{B}(0, r) \cap\left\{z: \boldsymbol{\delta}_{\operatorname{gr}\left(Q_{i}\right)}(z)>2 \Gamma r^{i+1}\right\}\right)}{\boldsymbol{\alpha}(m) r^{m}}=0 .
$$

Therefore (2b) is a consequence of 3.12 Moreover, using 3.12 , it follows that $A$ is approximately differentiable of order $(l, \beta)$ whenever either $l<k$ and $0 \leq \beta \leq 1$ or $l=k$ and $0 \leq \beta \leq \alpha$. We prove now (2a), whose proof is slightly more involved. We fix $2 \leq i \leq k$ and we replace $\Gamma$ by a larger number, if necessary, in order to have

$$
\left|f_{i}^{-1}(w)-f_{i}^{-1}(z)\right| \leq \Gamma|w-z|, \quad\left|f_{i}(w)-f_{i}(z)\right| \leq \Gamma|w-z|
$$

for $w, z \in \mathbf{B}(0,4)$. Therefore we have

$$
\begin{gathered}
f_{i}[A \cap \mathbf{C}(T, z, \epsilon r / \Gamma, \epsilon r / \Gamma)] \subseteq A_{i} \cap \mathbf{C}(T, z, \epsilon r / \Gamma, 3 r), \\
\mathscr{H}^{m}\left(A_{i} \cap \mathbf{C}(T, z, \epsilon r / \Gamma, 3 r)\right) \geq \Gamma^{-m} \mathscr{H}^{m}(A \cap \mathbf{C}(T, z, \epsilon r / \Gamma, \epsilon r / \Gamma)),
\end{gathered}
$$

whenever $0<r \leq 1,0<\epsilon \leq 1$ and $z \in \mathbf{B}(0, r / \Gamma)$. We fix $0<\epsilon \leq 1$ and, using 3.11, we can find $0<\rho \leq 1$ and $\eta>0$ such that

$$
\begin{gathered}
\mathscr{H}^{m}\left(A_{i} \cap \mathbf{B}(0,5 r) \cap\left\{w:\left|T_{\natural}^{\perp}(w)-P_{i}\left(T_{\natural}(w)\right)\right|>\epsilon r^{i}\right\}\right)<\eta \Gamma^{-m} \boldsymbol{\alpha}(m) r^{m}, \\
\mathscr{H}^{m}(\mathbf{C}(T, z, \epsilon r / \Gamma, \epsilon r / \Gamma) \cap A) \geq 2 \eta \boldsymbol{\alpha}(m) r^{m} \quad \text { for every } z \in T \cap \mathbf{B}(0, r),
\end{gathered}
$$

whenever $0<r \leq \rho$. Let $0<r \leq \rho$ and $z \in \operatorname{gr}\left(P_{i}\right) \cap \mathbf{B}(0, r / \Gamma)$. Then

$$
\begin{aligned}
\mathbf{C}(T, z, \epsilon r / \Gamma, 3 r) \subseteq & \left(\mathbf{B}(0,5 r) \cap\left\{w:\left|P_{i}\left(T_{\natural}(w)\right)-T_{\natural}^{\perp}(w)\right|>\epsilon r^{i}\right\}\right) \\
& \cup \mathbf{C}\left(T, z, \epsilon r / \Gamma, 2 \epsilon r^{i}\right) .
\end{aligned}
$$

In fact if $w \in \mathbf{C}(T, z, \epsilon r / \Gamma, 3 r)$ and $\left|P_{i}\left(T_{\natural}(w)\right)-T_{\natural}^{\perp}(w)\right| \leq \epsilon r^{i}$ then

$$
\begin{aligned}
& \left|P_{i}\left(T_{\natural}(w)\right)-P_{i}\left(T_{\natural}(z)\right)\right| \\
& \quad=\left|\sum_{j=1}^{i}\left\langle\left(T_{\natural}(w-z)^{j} / j !\right) \odot\left(T_{\natural}(z)^{i-j} /(i-j) !\right), \mathrm{D}^{i} P(0)\right\rangle\right| \\
& \quad \leq i\left\|\mathrm{D}^{i} P(0)\right\| \Gamma^{-i} \epsilon r^{i} \leq \epsilon r^{i}
\end{aligned}
$$


and we infer

$$
\left|T_{\natural}^{\perp}(z)-T_{\natural}^{\perp}(w)\right| \leq 2 \epsilon r^{i}, \quad w \in \mathbf{C}\left(T, z, \epsilon r / \Gamma, 2 \epsilon r^{i}\right) .
$$

We can now conclude that

$$
\mathscr{H}^{m}\left(A_{i} \cap \mathbf{C}\left(T, z, \epsilon r / \Gamma, 2 \epsilon r^{i}\right)\right) \geq \Gamma^{-m} \eta \boldsymbol{\alpha}(m) r^{m}
$$

and (2a) is proved.

By 3.8(1) we immediately conclude that $\Theta_{*}^{m}\left(\mathscr{H}^{m}\llcorner A, 0)>0\right.$ and $T \subseteq$ $\operatorname{Tan}_{*}^{m}\left(\mathscr{H}^{m}\llcorner A, 0)\right.$. By (2b) and 3.6 we conclude that $\operatorname{Tan}^{* m}\left(\mathscr{H}^{m}\llcorner A, 0) \subseteq T\right.$. Finally let $R: T \rightarrow T^{\perp}$ be a polynomial function of degree at most $k$ such that $R(0)=0$ and $\mathrm{D} R(0)=0$ and satisfying 3.8(1) and 3.8(2). Let

$$
\begin{gathered}
R_{i}(\chi)=\left\langle\chi^{i} / i !, \mathrm{D}^{i} R(0)\right\rangle \quad \text { for } \chi \in T \text { and } i=1, \ldots, k, \\
B_{1}=A_{1}, \quad B_{i}=\left\{x-R_{i-1}\left(T_{\natural}(x)\right): x \in B_{i-1}\right\} \text { for } i=2, \ldots, k .
\end{gathered}
$$

We prove by induction that $P_{i}=R_{i}$ for $i=1, \ldots, k$. Assume, for $j=1, \ldots, i$ and $i<k$, that $P_{j}=R_{j}$ and observe that $A_{i+1}=B_{i+1}$. Let $\epsilon>0,0<\rho \leq 1$ and $\eta>0$ such that

$$
\begin{gathered}
\mathscr{H}^{m}\left(\mathbf{C}\left(T, z, \epsilon r, \epsilon r^{i+1}\right) \cap B_{i+1}\right) \geq \eta \boldsymbol{\alpha}(m) r^{m} \text { for every } z \in \mathbf{B}(0, r) \cap \operatorname{gr}\left(R_{i+1}\right), \\
\mathscr{H}^{m}\left(A_{i+1} \cap \mathbf{B}(0,2 r) \cap\left\{z: \boldsymbol{\delta}_{\operatorname{gr}\left(P_{i+1}\right)}(z)>\epsilon r^{i+1}\right\}\right) \leq(\eta / 2) \boldsymbol{\alpha}(m) r^{m}
\end{gathered}
$$

whenever $0<r \leq \rho$. Therefore for every $z \in \mathbf{B}(0, r) \cap \operatorname{gr}\left(R_{i+1}\right)$ and for every $0<r \leq \rho$ we conclude that

$$
\mathscr{H}^{m}\left(B_{i+1} \cap \mathbf{C}\left(T, z, \epsilon r, \epsilon r^{i+1}\right) \cap\left\{z: \boldsymbol{\delta}_{\operatorname{gr}\left(P_{i+1}\right)}(z) \leq \epsilon r^{i+1}\right\}\right) \geq(\eta / 2) \boldsymbol{\alpha}(m) r^{m}
$$

and $P_{i+1}=R_{i+1}$ by 3.13 .

3.15 Remark. A conceptually similar characterization has been proved for the notion of pointwise differentiability in [Men16, 3.22]. Moreover the reader may find useful to compare 3.14(2) and [AS94, 3.4], where a concept of approximate tangent paraboloid is introduced by means of inhomogeneous dilations and weak convergence of Radon measures.

3.16 Remark. Suppose $A \subseteq \mathbf{R}^{n}$ and $a \in \mathbf{R}^{n}$. It is not difficult to see that the condition

$$
\operatorname{Tan}^{m}\left(\mathscr{H}^{m}\llcorner A, a) \in \mathbf{G}(n, m) \text { for some integer } 1 \leq m \leq n\right.
$$

is necessary and sufficient to conclude that $A$ is approximately differentiable of order 1 at $a$. In fact the necessity is asserted in 3.14 while the sufficiency follows from 3.10 and 3.6

3.17 Remark. We describe now a simple example which illustrates some features of the notion of approximate differentiability of order 1 .

With each $\gamma>1$ and $\gamma^{-1}<\alpha<(\gamma-1)^{-1}$ we associate the family $F_{\alpha, \gamma}$ consisting of the subsets

$$
\mathbf{R}^{2} \cap\left(\left\{\left(n^{-\alpha}, t\right): 0 \leq t \leq n^{-\alpha \gamma}\right\} \cup\left\{\left(-n^{-\alpha}, t\right): 0 \leq t \leq n^{-\alpha \gamma}\right\}\right)
$$

correspoding to the integers $n \geq 1$. We define

$$
A_{\alpha, \gamma}=\left(\mathbf{R}^{2} \cap\{(s, 0):-1 \leq s \leq 1\}\right) \cup \bigcup F_{\alpha, \gamma} .
$$


Since $\alpha \gamma>1$ then $\mathscr{H}^{1}\left(A_{\alpha, \gamma}\right)<\infty$. Moreover, for each $n \geq 1$,

$(n-1)^{\alpha} \sum_{i=n}^{\infty} i^{-\alpha \gamma} \geq(n-1)^{\alpha} \int_{n}^{\infty} x^{-\alpha \gamma} d \mathscr{L}^{1} x=(n-1)^{\alpha}(\alpha \gamma-1)^{-1} n^{1-\alpha \gamma} \rightarrow \infty$

as $n \rightarrow \infty$. Therefore $\boldsymbol{\Theta}_{*}^{1}\left(\mathscr{H}^{1}\left\llcorner A_{\alpha, \gamma}, 0\right)=\infty\right.$. Finally $A_{\alpha, \gamma}$ is approximately differentiable of order 1 at 0 by 3.16 since

$$
\operatorname{Tan}^{1}\left(\mathscr{H}^{1}\left\llcorner A_{\alpha, \gamma}, 0\right)=\mathbf{R} \times\{0\} .\right.
$$

3.18 Remark. Let $A \subseteq \mathbf{R}^{n}, a \in \mathbf{R}^{n}$ and let $0 \leq \mu \leq \nu$ be integers. Since

$$
\operatorname{Tan}^{* \nu}\left(\mathscr { H } ^ { \nu } \llcorner A , a ) \subseteq \operatorname { T a n } ^ { * \mu } \left(\mathscr{H}^{\mu}\llcorner A, a),\right.\right.
$$

we deduce by 3.14 that the integer $m$ in 3.8 is uniquely determined by $A$ and $a$.

3.19 Definition. Let $A \subseteq \mathbf{R}^{n}$ and $a \in \mathbf{R}^{n}$. Suppose $A$ is approximately differentiable of order 1 at $a$ and $m$ and $T$ are as in 3.8. We define the approximate tangent space of $A$ at $a$ to be the $m$ dimensional subspace $T$ and we denote it by ap $\operatorname{Tan}(A, a)$. Moreover we define the approximate normal space of $A$ at $a$ to be

$$
\text { ap } \operatorname{Nor}(A, a)=\mathbf{R}^{n} \cap\{v: v \bullet u=0\} .
$$

3.20 Definition. Let $A \subseteq \mathbf{R}^{n}$, let $k \geq 2$ be an integer and $a \in \mathbf{R}^{n}$. If $A$ is approximately differentiable of order $k$ at $a$ then we define the approximate differential of order $k$ of $A$ at $a$ to be the symmetric $k$ linear map

$$
\text { ap } \mathrm{D}^{k} A(a)=\mathrm{D}^{k}\left(P \circ T_{\natural}\right)(0) \in \bigodot^{k}\left(\mathbf{R}^{n}, \mathbf{R}^{n}\right),
$$

where $T=\operatorname{ap} \operatorname{Tan}(A, a)$ and $P: T \rightarrow T^{\perp}$ is as in 3.8 .

3.21 Remark. Suppose $A \subseteq \mathbf{R}^{n}$.

Following [Sim83, 11.2, 11.4] (see also [FM99, 2.2]) we consider the map $P_{A}$ whose domain is given by the set of $a \in \mathbf{R}^{n}$ such that there exist an integer $1 \leq m \leq n, T \in \mathbf{G}(n, m)$ and $0<\theta<\infty$ such that

$$
\lim _{r \rightarrow 0+} r^{-m} \int_{A} f((x-a) / r) d \mathscr{H}^{m} x=\theta \int_{T} f d \mathscr{H}^{m} \quad \text { whenever } f \in \mathscr{K}\left(\mathbf{R}^{n}\right),
$$

and whose value $P_{A}(a)$ at $a$ equals $T$. In fact, one may readily verifies that $m$, $T$ and $\theta$ are uniquely determined by $A$ and $a$.

Then it is not difficult to check that if $a \in \operatorname{dmn} P_{A}$ and $m=\operatorname{dim} P_{A}(a)$ then

$$
\begin{gathered}
P_{A}(a) \subseteq \operatorname{Tan}_{*}^{m}\left(\mathscr{H}^{m}\llcorner A, a), \quad \operatorname{Tan}^{* m}\left(\mathscr{H}^{m}\llcorner A, a) \subseteq P_{A}(a),\right.\right. \\
\Theta^{m}\left(\mathscr{H}^{m}\llcorner A, a)=\theta .\right.
\end{gathered}
$$

Using 3.16 and 3.14 we deduce

$$
\operatorname{dmn} P_{A} \subseteq \mathrm{dmn} \operatorname{ap} \operatorname{Tan}(A, \cdot), \quad P_{A}(a)=\operatorname{ap} \operatorname{Tan}(A, a) \text { whenever } a \in \operatorname{dmn} P_{A} .
$$

If $A_{\alpha, \gamma}$ is defined as in 3.17 then $0 \in\left(\operatorname{dmn} \operatorname{ap} \operatorname{Tan}\left(A_{\alpha, \gamma}, \cdot\right)\right) \sim\left(\operatorname{dmn} P_{A_{\alpha, \gamma}}\right)$. 
3.22 Remark. Let $1 \leq m \leq n$ and $k \geq 1$ be integers, $0 \leq \alpha \leq 1, A \subseteq \mathbf{R}^{n}$, $B \subseteq \mathbf{R}^{n}$ and $a \in \mathbf{R}^{n}$. Suppose $A$ is approximately differentiable of order $(k, \alpha)$ at $a, m=\operatorname{dim} \operatorname{ap} \operatorname{Tan}(A, a)$ and

$$
\Theta^{m}\left(\mathscr{H}^{m}\llcorner A \sim B, a)=0, \quad \boldsymbol{\Theta}^{m}\left(\mathscr{H}^{m}\llcorner B \sim A, a)=0 .\right.\right.
$$

Then $B$ is approximately differentiable of order $(k, \alpha)$ at $a$ with

$$
\begin{gathered}
\operatorname{ap} \operatorname{Tan}(A, a)=\operatorname{ap} \operatorname{Tan}(B, a), \\
\operatorname{apD}^{i} A(a)=\operatorname{apD} D^{i} B(a) \text { for } i=2, \ldots, k .
\end{gathered}
$$

3.23 Theorem. Let $1 \leq m \leq n$ and $k \geq 1$ be integers, $0 \leq \alpha \leq 1$ and let $A \subseteq \mathbf{R}^{n}$ be $\mathscr{H}^{m}$ measurable and $\left(\mathscr{H}^{m}, m\right)$ rectifiable of class $(k, \alpha)$.

Then for $\mathscr{H}^{m}$ a.e. $a \in A$ the set $A$ is approximately differentiable of order $(k, \alpha)$ at a with

$$
\operatorname{ap} \operatorname{Tan}(A, a) \in \mathbf{G}(n, m)
$$

Proof. Since an $m$ dimensional submanifold $M$ of class $(k, \alpha)$ of $\mathbf{R}^{n}$ locally corresponds at each $a \in M$ to a graph of function $f: \operatorname{Tan}(M, a) \rightarrow \operatorname{Nor}(M, a)$ of class $(k, \alpha)$ with $\mathrm{D} f\left(\operatorname{Tan}(M, a)_{\mathfrak{t}}(a)\right)=0$, one readily checks that $M$ is approximately differentiable of order $(k, \alpha)$ at each of its points. Then the conclusion follows from [Fed69, 2.10.19(4)] and 3.22

3.24 Remark. The conclusion of 3.23 may not hold if we replace " $\left(\mathscr{H}^{m}, m\right)$ rectifiable" with "countably $\left(\mathscr{H}^{m}, m\right)$ rectifiable", as the following example in $\mathbf{R}^{2}$ shows,

$$
\bigcup_{n=1}^{\infty}\left\{\left(n^{-1}, t\right): 0 \leq t \leq 1\right\} \cup\{(0, t): 0 \leq t \leq 1\} .
$$

3.25 Theorem. Let $1 \leq m \leq n$ be integers, let $A \subseteq \mathbf{R}^{n}$ be $\mathscr{H}^{m}$ measurable and $\left(\mathscr{H}^{m}, m\right)$ rectifiable of class 1 and let $\nu: A \rightarrow \mathbf{R}^{n}$ be a map such that for $\mathscr{H}^{m}$ a.e. $x \in A$ there exists $0 \leq \lambda<\infty$ such that

$$
\Theta^{m}\left(\mathscr{H}^{m}\llcorner A \cap\{z:|\nu(z)-\nu(x)|>\lambda|z-x|\}, x)=0 .\right.
$$

Then $\nu$ is $\mathscr{H}^{m}\left\llcorner A\right.$ measurable and $\left(\mathscr{H}^{m}\llcorner A, m)\right.$ approximately differentiable 13 at $\mathscr{H}^{m}$ a.e. $x \in A$.

If additionally $\nu(x) \in \operatorname{ap} \operatorname{Nor}(A, x)$ for $\mathscr{H}^{m}$ a.e. $x \in A$ and $A$ is $\left(\mathscr{H}^{m}, m\right)$ rectifiable of class 2 then

$$
\left(\mathscr{H}^{m}\llcorner A, m) \text { ap } \mathrm{D} \nu(x)(u) \bullet v=-\operatorname{ap} \mathrm{D}^{2} A(x)(u, v) \bullet \nu(x)\right.
$$

for every $u, v \in \operatorname{ap} \operatorname{Tan}(A, x)$ and for $\mathscr{H}^{m}$ a.e. $x \in A$.

Proof. By Fed69, 3.2.29, 3.1.19(4), 2.10.19(4), 3.2.16] it is enough to prove the statement in the following special case: let $U \subseteq \mathbf{R}^{n}, V \subseteq \mathbf{R}^{m}$ be bounded open sets and let $\phi: U \rightarrow \mathbf{R}^{m}, \psi: V \rightarrow \mathbf{R}^{n}$ be maps of class 1 (of class 2 if $A$ is $\left(\mathscr{H}^{m}, m\right)$ rectifiable of class 2) such that $A \subseteq \operatorname{im} \psi$ and $\phi \circ \psi=\mathbf{1}_{V}$. Let $M=\operatorname{im} \psi$ and observe that $\phi \mid M=\psi^{-1}, \phi[A]$ is an $\mathscr{H}^{m}$ measurable subset of $\mathbf{R}^{m}$. Moreover we can prove that $\nu$ is $\mathscr{H}^{m}\llcorner A$ measurable by [Fed69, 2.9.13]. In fact one verifies that $V=\left\{(a, \mathbf{B}(a, r)): a \in \mathbf{R}^{n}, 0<r<\infty\right\}$ is an $\mathscr{H}^{m}\llcorner A$ Vitali relation by [Fed69, 2.8.18] and, since $\boldsymbol{\Theta}^{m}\left(\mathscr{H}^{m}\llcorner A, x)=1\right.$ for $\mathscr{H}^{m}$ a.e.

\footnotetext{
${ }^{13}$ See [Fed69, 3.2.16].
} 
$x \in A$ by Fed69, 3.2.19], we conclude that $\nu$ is $\left(\mathscr{H}^{m}\llcorner A, V)\right.$ approximately continuous 14 at $\mathscr{H}^{m}$ a.e. $x \in A$.

Let $\eta=\nu \circ(\psi \mid \phi[A])$ and, by [Fed69, 2.9.11], we deduce that $\eta$ is approximately differentiable of order $(0,1)$ at $\mathscr{L}^{m}$ a.e. $\chi \in \phi[A]$. Therefore by 2.11(3) there exist countably many maps $\eta_{j}: \mathbf{R}^{m} \rightarrow \mathbf{R}^{n}$ of class 1 such that

$$
\mathscr{L}^{m}\left(\phi[A] \sim \bigcup_{j=1}^{\infty}\left\{\chi: \eta_{j}(\chi)=\eta(\chi)\right\}\right)=0 .
$$

We deduce, by $\left[\right.$ Fed69, 2.10.19(4)], that $\nu$ is $\left(\mathscr{H}^{m}\llcorner A, m)\right.$ approximately differentiable at $\mathscr{H}^{m}$ a.e. $x \in A$ because

$$
\mathscr{H}^{m}\left(A \sim \bigcup_{j=1}^{\infty}\left\{x:\left(\eta_{j} \circ \phi\right)(x)=\nu(x)\right\}\right)=0 .
$$

If we further assume $\nu(x) \in \operatorname{ap} \operatorname{Nor}(A, x)$ for $\mathscr{H}^{m}$ a.e. $x \in A$ and $A$ is $\left(\mathscr{H}^{m}, m\right)$ rectifiable of class 2 then, for every $j \geq 1$, we define

$$
\nu_{j}(x)=\left(\operatorname{Nor}(M, x)_{\natural} \circ \eta_{j} \circ \phi\right)(x) \text { for } x \in M,
$$

we observe that $\nu_{j}$ is of class 1 relative to $M$ and, by [Fed69, 2.10.19(4)] and 3.22.

$$
\mathscr{H}^{m}\left(A \sim \bigcup_{j=1}^{\infty}\left\{x: \nu_{j}(x)=\nu(x)\right\}\right)=0 .
$$

Since, by [Fed69, 2.10.19(4), 3.2.16] and 3.22

$$
\mathrm{D} \nu_{j}(x)(u) \bullet v=-\operatorname{apD}^{2} A(x)(u, v) \bullet \nu_{j}(x) \quad \text { for every } u, v \in \operatorname{ap} \operatorname{Tan}(A, x)
$$

and $\left(\mathscr{H}^{m}\llcorner A, m)\right.$ ap $\mathrm{D} \nu(x)=\mathrm{D} \nu_{j}(x)$ for $\mathscr{H}^{m}$ a.e. $x \in A$, the conclusion follows.

3.26 Remark. The conclusion of the second part of 3.25 may fail to hold at $\mathscr{H}^{m}$ a.e. $a \in A$ if we omit the hypothesis " $A$ is $\left(\mathscr{H}^{m}, m\right)$ rectifiable of class 2 ", even if we assume that $A$ is an $m$ dimensional submanifold of class 1 . This fact can be easily deduced from Koh77, and 5.6.

Moreover the same conclusion may fail to hold at $\mathscr{H}^{m}$ a.e. $a \in A$ if we omit the hypothesis " $A$ is $\left(\mathscr{H}^{m}, m\right)$ rectifiable of class 2 " but we assume $\nu(x)=\zeta$ for $\mathscr{H}^{m}$ a.e. $x \in A$ for some $\zeta \in \mathbf{S}^{n-1}$. In fact it is proved in [AS94, Appendix] that for every $0<\alpha<1$ there exists a function $f: \mathbf{R} \rightarrow \mathbf{R}$ of class $(1, \alpha)$ and a Cantor-type set $E \subseteq \mathbf{R}$ such that

$$
\begin{gathered}
\mathscr{L}^{1}(E)>0, \quad \mathrm{D} f(x)=0 \quad \text { for every } x \in E, \\
\mathscr{L}^{1}(E \cap\{x: f(x)=g(x)\})=0 \quad \text { whenever } g: \mathbf{R} \rightarrow \mathbf{R} \text { is of class } 2 .
\end{gathered}
$$

If $A=\operatorname{gr}(f \mid E)$ then, by , $\mathscr{H}^{1}\left(A \cap \operatorname{dmn} \operatorname{apD}^{2} A\right)=0$.

\section{Relation with pointwise differentiability}

4.1 Definition. Suppose $X$ is a normed vector space, $B \subseteq X$ and $a \in X$.

We define the upper tangent cone of $B$ at a by

$$
\operatorname{Tan}^{*}(B, a)=X \cap\left\{v: \liminf _{r \rightarrow 0+} r^{-1} \boldsymbol{\delta}_{B}(a+r v)=0\right\}
$$

\footnotetext{
${ }^{14}$ See [Fed69, 2.9.12].
} 
and the lower tangent cone of $B$ at a by

$$
\operatorname{Tan}_{*}(B, a)=X \cap\left\{v: \lim _{r \rightarrow 0+} r^{-1} \boldsymbol{\delta}_{B}(a+r v)=0\right\}
$$

In case $\operatorname{Tan}_{*}(B, a)=\operatorname{Tan}^{*}(B, a)$, this set is denoted by $\operatorname{Tan}(B, a)$ and we call it the tangent cone of $B$ at a. Finally the [lower, upper] normal cone of $B$ at $a$ is defined to be the set of $v \in \mathbf{R}^{n}$ such that $v \bullet u \leq 0$ whenever $u \in\left[\operatorname{Tan}_{*}(A, a), \operatorname{Tan}^{*}(A, a)\right] \operatorname{Tan}(A, a)$, and it is denoted by

$$
\left[\operatorname{Nor}_{*}(A, a), \operatorname{Nor}^{*}(A, a)\right] \operatorname{Nor}(A, a) .
$$

4.2 Remark. If $1 \leq m \leq n$ are integers and $B \subseteq \mathbf{R}^{n}$ then one may verify that

$$
\begin{gathered}
\operatorname{Tan}^{* m}\left(\mathscr{H}^{m}\llcorner B, a) \subseteq \operatorname{Tan}^{*}(B, a)\right. \\
\cup । \\
\operatorname{Tan}_{*}^{m}\left(\mathscr{H}^{m}\llcorner B, a) \subseteq \operatorname{Tan}_{*}(B, a) .\right.
\end{gathered}
$$

Moreover one may readily verify that $\operatorname{Tan}_{*}(B, a)$ and $\operatorname{Tan}^{*}(B, a)$ are closed cones.

4.3 Remark. This notation does not agree with [Fed59, 4.3], [Fed69, 3.1.21] and Men16. In fact $\operatorname{Tan}^{*}(B, a)$ is denoted by $\operatorname{Tan}(B, a)$ therein.

4.4 Remark. Employing [Fed59, 4.1], we observe that if $A$ is a closed subset of $\mathbf{R}^{n}, a \in A$ and $\operatorname{reach}(A, a)>0$ then, by [Fed59, 4.8(10),(12)],

$$
\operatorname{Tan}_{*}(A, a)=\operatorname{Tan}^{*}(A, a) .
$$

4.5 Definition. Let $k$ and $n$ be positive integers, $0 \leq \alpha \leq 1$ and $B \subseteq \mathbf{R}^{n}$. We say that $B$ is pointwise differentiable of order $(k, \alpha)$ at a if there exists a submanifold $M \subseteq \mathbf{R}^{n}$ of class $(k, \alpha)$ such that $a \in M$,

$$
\begin{gathered}
\lim _{r \rightarrow 0} r^{-1} \sup \left\{\left|\boldsymbol{\delta}_{M}(x)-\boldsymbol{\delta}_{B}(x)\right|: x \in \mathbf{B}(a, r)\right\}=0, \\
\lim _{r \rightarrow 0} r^{-k} \sup \left\{\boldsymbol{\delta}_{M}(x): x \in \mathbf{B}(a, r) \cap B\right\}=0 \quad \text { if } \alpha=0, \\
\limsup _{r \rightarrow 0} r^{-k-\alpha} \sup \left\{\boldsymbol{\delta}_{M}(x): x \in \mathbf{B}(a, r) \cap B\right\}<\infty \quad \text { if } \alpha>0 .
\end{gathered}
$$

4.6 Remark. This concept has been introduced in [Men16, 3.3]. In4.11 and 4.12 we employ the concept of pointwise differential of order $i$ for sets, introduced in [Men16, 3.12].

4.7 Remark. It is worth to mention that, for sets, pointwise differentiability does not imply approximate differentiability. In fact, suppose $n \geq 1$ is an integer and $B$ is a countable dense subset of $\mathbf{R}^{n}$. Then for every integer $k \geq 1$ the set $B$ is pointwise differentiable of order $k$ at every $x \in \mathbf{R}^{n}$. But $B$ is not approximately differentiable of order 1 at every $x \in \mathbf{R}^{n}$.

4.8 Lemma. Let $B \subseteq \mathbf{R}^{n}$ and $a \in \operatorname{Clos} B$.

Then the following statements hold.

(1) If $M=\left\{a+v: v \in \operatorname{Tan}^{*}(B, a)\right\}$ then

$$
\lim _{r \rightarrow 0} r^{-1} \sup \left\{\boldsymbol{\delta}_{M}(x): x \in \mathbf{B}(a, r) \cap B\right\}=0 .
$$


(2) If $M=\left\{a+v: v \in \operatorname{Tan}_{*}(B, a)\right\}$ then

$$
\lim _{r \rightarrow 0} r^{-1} \sup \left\{\boldsymbol{\delta}_{B}(x): x \in \mathbf{B}(a, r) \cap M\right\}=0 .
$$

(3) The condition

$$
\operatorname{Tan}(B, a) \in \mathbf{G}(n, m) \quad \text { for some integer } 0 \leq m \leq n
$$

is necessary and sufficient to conclude that $A$ is pointwise differentiable of order 1 at $a$.

Proof. Proof of (11). If there existed $\epsilon>0, r_{i}>0, r_{i} \rightarrow 0$ as $i \rightarrow \infty$ and $x_{i} \in B \cap \mathbf{B}\left(a, r_{i}\right)$ such that $\boldsymbol{\delta}_{M}\left(x_{i}\right) \geq \epsilon r_{i}$ then, possibly passing to a subsequence, we could assume there would exist $v \in \mathbf{S}^{n-1}$ such that $\left(x_{i}-a\right) /\left|x_{i}-a\right| \rightarrow v$ as $i \rightarrow \infty$. Then $v \in \operatorname{Tan}^{*}(B, a)$,

$$
\epsilon \leq r_{i}^{-1}\left|x_{i}-a-\right| x_{i}-a|v| \leq\left|x_{i}-a\right|^{-1}\left|x_{i}-a-\right| x_{i}-a|v| \text { for } i \geq 1
$$

and we would get a contradiction.

Proof of (2). Suppose $\epsilon>0$ and observe there exist an integer $l \geq 1$, $v_{1}, \ldots, v_{l} \in \operatorname{Tan}_{*}(B, a) \cap \mathbf{S}^{n-1}$ and $\eta>0$ such that $r^{-1} \boldsymbol{\delta}_{B}\left(a+r v_{i}\right)<\epsilon$ whenever $i=1, \ldots, l$ and $0<r \leq \eta$ and

$$
\operatorname{Tan}_{*}(B, a) \cap \mathbf{S}^{n-1} \subseteq \bigcup_{i=1}^{l} \mathbf{B}\left(v_{i}, \epsilon\right) .
$$

If $0<r \leq \eta$ and $v \in \mathbf{B}(0, r) \cap \operatorname{Tan}_{*}(B, a) \sim\{0\}$ then we choose $i=1, \ldots, l$ such that $\left|(v /|v|)-v_{i}\right| \leq \epsilon$ and, since $\operatorname{Lip} \boldsymbol{\delta}_{B} \leq 1$, we conclude that $\boldsymbol{\delta}_{B}(a+v) \leq 2 \epsilon|v|$.

Proof of (3). For the necessity, suppose $M$ is as in 4.5 when $k=1$ and $\alpha=0$, observe that $\operatorname{Tan}(M, a)=\operatorname{Tan}^{*}(B, a)$ by $[M e n 16,3.4]$ and $\operatorname{Tan}(M, a) \subseteq$ $\operatorname{Tan}_{*}(B, a)$ because

$$
\lim _{\operatorname{Tan}(M, a) \ni v \rightarrow 0}|v|^{-1} \boldsymbol{\delta}_{M}(a+v)=0 .
$$

For the sufficiency let $M=\{a+v: v \in \operatorname{Tan}(B, a)\}$ and, since $a \in \operatorname{Clos} B$, one verifies that

$$
\begin{gathered}
\sup \left\{\left|\boldsymbol{\delta}_{B}(x)-\boldsymbol{\delta}_{M}(x)\right|: x \in \mathbf{B}(a, r)\right\} \leq \\
\leq \sup \left(\left\{\boldsymbol{\delta}_{B}(x): x \in \mathbf{B}(a, 2 r) \cap M\right\} \cup\left\{\boldsymbol{\delta}_{M}(x): x \in \mathbf{B}(a, 2 r) \cap B\right\}\right),
\end{gathered}
$$

Therefore the conclusion comes from (11) and (2).

4.9 Remark. Compare 4.8(3) with the analogous result for approximate differentiability in 3.16. Moreover 4.8(3) is a restatement of [Men16, 3.19].

4.10 Remark. If $M$ is an $m$ dimensional submanifold of class 1 of $\mathbf{R}^{n}$ then, by 4.8(3), 3.16 and 4.2, one may readily infer that

$$
\operatorname{Tan}(M, a)=\operatorname{Tan}^{m}\left(\mathscr{H}^{m}\llcorner M, a) \quad \text { for every } a \in M .\right.
$$

4.11 Theorem. Let $1 \leq m \leq n$ and $k \geq 1$ be integers, $0 \leq \alpha \leq 1, A \subseteq \mathbf{R}^{n}$ and $a \in \mathbf{R}^{n}$. Suppose $A$ is approximately differentiable of order $(k, \alpha)$ at $a$ and $m=\operatorname{dim} \operatorname{ap} \operatorname{Tan}(A, a)$.

Then there exists $B \subseteq A$ pointwise differentiable of order $(k, \alpha)$ at a such that

$$
\begin{gathered}
\Theta^{m}\left(\mathscr{H}^{m}\llcorner A \sim B, a)=0,\right. \\
\operatorname{ap} \operatorname{Tan}(A, a)=\operatorname{Tan}(B, a)=\operatorname{Tan}^{m}\left(\mathscr{H}^{m}\llcorner B, a),\right. \\
\operatorname{ptD}^{i} B(a, \operatorname{Tan}(B, a))=\operatorname{apD}^{i} A(a) \quad \text { for } i=2, \ldots, k .
\end{gathered}
$$


Proof. Assume $a=0$ and suppose $T=\operatorname{ap} \operatorname{Tan}(A, 0), P: T \rightarrow T^{\perp}$ is defined by

$$
P(\chi)=\sum_{j=2}^{k}\left\langle\chi^{j} / j !, \text { ap } \mathrm{D}^{j} A(0)\right\rangle \quad \text { for } \chi \in T
$$

and $\Gamma=\sup \left\{1, \sum_{j=2}^{k}\left\|\operatorname{ap} D^{j} A(0)\right\| / j !\right\}$. In particular if $k=1$ then $P=0$ and $\Gamma=1$. By 3.11 and 3.7 we infer that

$$
\begin{gathered}
\boldsymbol{\Theta}^{m}\left(\mathscr{H}^{m}\left\llcorner A \sim \mathbf{X}_{k}(0, T, P, \epsilon), a\right)=0 \quad \text { for every } \epsilon>0 \text { if } \alpha=0,\right. \\
\boldsymbol{\Theta}^{m}\left(\mathscr{H}^{m}\left\llcorner A \sim \mathbf{X}_{k, \alpha}(0, T, P, \lambda), a\right)=0 \quad \text { for some } 0 \leq \lambda<\infty \text { if } \alpha>0 .\right.
\end{gathered}
$$

We fix $0 \leq \lambda<\infty$ as above if $\alpha>0$. We define, for every integer $i \geq 1$,

$$
A_{i}=A \cap \mathbf{X}_{k}\left(0, T, P,(2 i)^{-1}\right) \text { if } \alpha=0, \quad A_{i}=A \cap \mathbf{X}_{k, \alpha}(0, T, P, \lambda) \text { if } \alpha>0 .
$$

Let $Q_{r}=\mathbf{R}^{n} \cap\left\{z:\left|T_{\natural}^{\perp}(z)\right| \leq r,\left|T_{\natural}(z)\right| \leq r\right\}$ for $0<r<\infty$. For every integer $i \geq 1$ let $\delta_{i}>0$ be such that

$$
\mathscr{H}^{m}\left(A \cap Q_{r} \sim A_{i}\right) \leq 2^{-i} \boldsymbol{\alpha}(m) r^{m} \quad \text { whenever } 0<r \leq \delta_{i}
$$

and we assume $\delta_{i+1}<\delta_{i}, \delta_{i} \rightarrow 0$ as $i \rightarrow \infty$,

$$
\delta_{1} \leq(2 \Gamma)^{-1} \text { if } \alpha=0, \quad \delta_{1} \leq(\lambda+\Gamma)^{-1 / \alpha} \text { if } \alpha>0 .
$$

We define, for every integer $i \geq 1$,

$$
C_{i}=T_{\natural}^{-1}\left[\mathbf{B}\left(0, \delta_{i}\right) \sim \mathbf{B}\left(0, \delta_{i+1}\right)\right], \quad B=\bigcup_{j=1}^{\infty} A_{j} \cap C_{j} .
$$

Observe that $B \subseteq \mathbf{X}(0, T, 1)$ and

$$
\left(Q_{\delta_{j}} \sim Q_{\delta_{j+1}}\right) \cap \mathbf{X}(0, T, 1) \sim C_{j}=\varnothing \quad \text { whenever } j \geq 1 .
$$

We can prove now that $\boldsymbol{\Theta}^{m}\left(\mathscr{H}^{m}\llcorner A \sim B, 0)=0\right.$. In fact, by 3.6 and 3.14 we infer $\boldsymbol{\Theta}^{m}\left(\mathscr{H}^{m}\llcorner A \sim \mathbf{X}(0, T, 1), 0)=0\right.$. Moreover, if $0<r \leq \delta_{1}$ and $i \geq 1$ are such that $\delta_{i+1}<r \leq \delta_{i}$ then

$$
\begin{gathered}
Q_{r} \cap A \cap \mathbf{X}(0, T, 1) \sim B \subseteq\left(Q_{r} \cap A \sim A_{i}\right) \cup \bigcup_{j=i+1}^{\infty} Q_{\delta_{j}} \cap A \sim A_{j}, \\
\mathscr{H}^{m}\left(Q_{r} \cap A \cap \mathbf{X}(0, T, 1) \sim B\right) \leq \boldsymbol{\alpha}(m) r^{m} \sum_{j=i}^{\infty} 2^{-j} .
\end{gathered}
$$

Since this implies $0 \in \operatorname{Clos} B$ by 3.14 , it follows that

$$
\begin{gathered}
\lim _{r \rightarrow 0} r^{-k} \sup \left\{\left|P\left(T_{\natural}(z)\right)-T_{\natural}^{\perp}(z)\right|: z \in B \cap T_{\natural}^{-1}[\mathbf{B}(0, r)]\right\}=0 \quad \text { if } \alpha=0, \\
\limsup _{r \rightarrow 0} r^{-k-\alpha} \sup \left\{\left|P\left(T_{\natural}(z)\right)-T_{\natural}^{\perp}(z)\right|: z \in B \cap T_{\natural}^{-1}[\mathbf{B}(0, r)]\right\} \leq \lambda \quad \text { if } \alpha>0 .
\end{gathered}
$$

In particular, $\operatorname{Tan}^{*}(B, 0) \subseteq T$. By 3.5 and 3.14 we get that

$$
T=\operatorname{Tan}_{*}^{m}\left(\mathscr { H } ^ { m } \llcorner A , 0 ) \subseteq \operatorname { T a n } _ { * } ^ { m } \left(\mathscr{H}^{m}\llcorner B, 0) .\right.\right.
$$

Therefore $B$ is pointwise differentiable of order 1 at $a$ with $T=\operatorname{Tan}(B, 0)=$ $\operatorname{Tan}^{m}\left(\mathscr{H}^{m}\llcorner B, 0)\right.$ by 4.2 and 4.8(3). Moreover, since $\operatorname{Tan}(\operatorname{gr} P, 0)=T$, we can use 3.11 to check that the conditions in 4.5 hold with $M$ replaced by gr $P$. Therefore, by [Men16, 3.12] and 3.20, we conclude that

$$
\operatorname{ptD}^{i} B(0, T)=\operatorname{apD}^{i} A(0) \text { for } i=2, \ldots, k .
$$


4.12 Theorem. Let $A \subseteq \mathbf{R}^{n}, a \in \mathbf{R}^{n}, \nu \in \mathbf{S}^{n-1}, 0<r<\infty$ and suppose

$$
\mathbf{U}(a+r \nu, r) \cap A=\varnothing .
$$

Then the following three statements hold.

(1) If $A$ is a submanifold of class 2 and $a \in A$ then

$$
\mathbf{b}_{A}(a)(v, v) \bullet \nu \leq r^{-1}|v|^{2} \quad \text { whenever } v \in \operatorname{Tan}(A, a) .
$$

(2) If $A$ is pointwise differentiable of order 2 at a then

$$
\operatorname{pt} \mathrm{D}^{2} A(a, \operatorname{Tan}(A, a))(v, v) \bullet \nu \leq r^{-1}|v|^{2} \quad \text { whenever } v \in \operatorname{Tan}(A, a) .
$$

(3) If $A$ is approximately differentiable of order 2 at a then

$$
\operatorname{ap} \mathrm{D}^{2} A(a)(v, v) \bullet \nu \leq r^{-1}|v|^{2} \quad \text { whenever } v \in \operatorname{ap} \operatorname{Tan}(A, a)
$$

Proof. Assume $a=0$. Observe that $\nu \in \operatorname{Nor}^{*}(A, 0)$.

The statement in (1) is classical. We give a proof here for completeness. If $T=\operatorname{Tan}(A, 0)$ then there exist a function $f: T \rightarrow T^{\perp}$ of class 2 and an open neighbourhood $U$ of $0 \in \mathbf{R}^{n}$ such that $\mathrm{D} f(0)=0, T_{\natural}[U]=T_{\natural}[U \cap A]$ and $A \cap U=\left\{\chi+f(\chi): \chi \in T_{\natural}[U]\right\}$. Since for every $\chi \in T_{\natural}[U]$

$$
|\chi+f(\chi)-r \nu| \geq r, \quad 2 r f(\chi) \bullet \nu \leq|\chi|^{2}+|f(\chi)|^{2},
$$

we conclude that $\mathrm{D}^{2} f(0)(v, v) \bullet \nu \leq r^{-1}|v|^{2}$ for every $v \in \operatorname{Tan}(A, 0)$ and, since $\mathbf{b}_{A}(0)=\mathrm{D}^{2} f(0)$, the statement in (1) follows.

The statement in (2) is mainly a consequence of [Men16, 3.18]. In fact suppose $T=\operatorname{Tan}(A, 0), P: T \rightarrow T^{\perp}$ is the homogeneous polynomial function of degree 2 such that pt $\mathrm{D}^{2} A(0, T)=\mathrm{D}^{2}\left(P \circ T_{\natural}\right)(0)$ (whose existence can be asserted, from instance, by Men16, 3.22]) and $B=\{\chi+P(\chi): \chi \in T\}$. If we prove that $\mathbf{U}(r \nu, r) \cap B=\varnothing$ then (2) is a consequence of (1). By contradiction let $x \in B \cap \mathbf{U}(r \nu, r)$ and, by [Men16, 3.18], for every positive integer $i$ we can select $x_{i} \in A$ such that

$$
\left|i T_{\natural}\left(x_{i}\right)+i^{2} T_{\natural}^{\perp}\left(x_{i}\right)-x\right| \rightarrow 0 \quad \text { as } i \rightarrow \infty .
$$

Since $\left|x_{i}-r \nu\right| \geq r$ for every $i \geq 1$, we get

$$
\begin{aligned}
& \left|i T_{\natural}\left(x_{i}\right)+i^{2} T_{\natural}^{\perp}\left(x_{i}\right)-r \nu\right|^{2} \\
& \quad=i^{2}\left|x_{i}-r \nu\right|^{2}+\left(i^{4}-i^{2}\right)\left|T_{\natural}^{\perp}\left(x_{i}\right)\right|^{2}+r^{2}-i^{2} r^{2} \\
& \quad \geq\left(i^{4}-i^{2}\right)\left|T_{\natural}^{\perp}\left(x_{i}\right)\right|^{2}+r^{2} \quad \text { for } i \geq 1 ;
\end{aligned}
$$

yet $\left|i T_{\natural}\left(x_{i}\right)+i^{2} T_{\natural}^{\perp}\left(x_{i}\right)-r \nu\right|<r$ for $i$ large. This is a contradiction.

Finally (3) is a consequence of (2) and 4.11. 


\section{$5 \quad$ Rectifiability and Borel measurability}

5.1 Lemma. Let $1 \leq m \leq n$ and $k \geq 1$ be integers, $0 \leq \alpha \leq 1, \gamma=k+\alpha$ and $A \subseteq \mathbf{R}^{n}$. Let $Y$ be the set of

$$
\left(a, T, \phi_{0}, \ldots, \phi_{k}\right) \in \mathbf{R}^{n} \times \mathbf{G}(n, m) \times \prod_{i=0}^{k} \bigodot^{i}\left(\mathbf{R}^{n}, \mathbf{R}^{n}\right)
$$

such that $\phi_{0}=T_{\natural}^{\perp}(a)$ and

$$
\lim _{r \rightarrow 0} \frac{\mathscr{H}^{m}\left(A \cap \mathbf{U}(a, r) \cap\left\{z:\left|T_{\natural}^{\perp}(z)-\sum_{j=0}^{k}\left\langle T_{\mathrm{\natural}}(z-a)^{j} / j !, \phi_{j}\right\rangle\right|>\lambda r^{\gamma}\right\}\right)}{\boldsymbol{\alpha}(m) r^{m}}=0
$$

for every $\lambda>0$ [for some $0 \leq \lambda<\infty$ ].

Then $Y$ is a Borel subset of $\mathbf{R}^{n} \times \mathbf{G}(n, m) \times \prod_{i=0}^{k} \bigodot^{i}\left(\mathbf{R}^{n}, \mathbf{R}^{n}\right)$.

Proof. Let $Z=\mathbf{R}^{n} \times \mathbf{G}(n, m) \times \prod_{j=0}^{k} \bigodot^{j}\left(\mathbf{R}^{n}, \mathbf{R}^{n}\right)$. If $0<\lambda<\infty, i \geq 1$ is an integer and $0<r<\infty$, we define $W_{\lambda, i, r}$ to be the set of $\left(a, T, \phi_{0}, \ldots, \phi_{k}\right) \in Z$ such that $\phi_{0}=T_{\natural}^{\perp}(a)$ and

$$
\mathscr{H}^{m}\left(A \cap \mathbf{U}(a, r) \cap\left\{z:\left|T_{\natural}^{\perp}(z)-\sum_{l=0}^{k}\left\langle T_{\natural}(z-a)^{l} / l !, \phi_{l}\right\rangle\right|>\lambda r^{\gamma}\right\}\right) \leq i^{-1} r^{m} .
$$

Then $W_{\lambda, i, r}$ is a closed subset of $Z$. In fact if $\left(a_{j}, T_{j}, \phi_{0, j}, \ldots, \phi_{k, j}\right) \in W_{\lambda, i, r}$, $j \geq 1$, is a sequence converging to $\left(a, T, \phi_{0}, \ldots, \phi_{k}\right) \in Z$ as $j \rightarrow \infty$, we define

$$
\begin{gathered}
P_{j}(\chi)=\sum_{l=0}^{k}\left\langle\left(\chi-T_{j}\left(a_{j}\right)\right)^{l} / l !, \phi_{l, j}\right\rangle \quad \text { for } \chi \in T_{j} \text { and } j \geq 1, \\
P(\chi)=\sum_{l=0}^{k}\left\langle\left(\chi-T_{\natural}(a)\right)^{l} / l !, \phi_{l}\right\rangle \quad \text { for } \chi \in T,
\end{gathered}
$$

and we observe that $P_{j}\left(T_{j}\right.$ घ $\left.(z)\right) \rightarrow P\left(T_{\natural}(z)\right)$ as $j \rightarrow \infty$, whenever $z \in \mathbf{R}^{n}$. Let

$$
\begin{gathered}
B_{j}=A \cap \mathbf{U}\left(a_{j}, r\right) \cap\left\{z:\left|T_{j \natural}^{\perp}(z)-P_{j}\left(T_{j \natural}(z)\right)\right|>\lambda r^{\gamma}\right\}, \\
B=A \cap \mathbf{U}(a, r) \cap\left\{z:\left|T_{\natural}^{\perp}(z)-P\left(T_{\natural}(z)\right)\right|>\lambda r^{\gamma}\right\}
\end{gathered}
$$

and observe that

$$
B \subseteq \bigcup_{j=1}^{\infty} \bigcap_{h=j}^{\infty} B_{h}
$$

Therefore, by [Fed69, 2.1.5(1)], we conclude that

$$
\mathscr{H}^{m}(B) \leq \lim _{j \rightarrow \infty} \mathscr{H}^{m}\left(\bigcap_{h=j}^{\infty} B_{h}\right) \leq \liminf _{j \rightarrow \infty} \mathscr{H}^{m}\left(B_{j}\right) \leq i^{-1} r^{m},
$$

$\left(a, T, \phi_{0}, \ldots, \phi_{k}\right) \in W_{\lambda, i, r}$ and $W_{\lambda, i, r}$ is closed.

Henceforth $Y$ is a Borel set because

$$
\begin{gathered}
Y=\bigcap_{l=1}^{\infty} \bigcap_{i=1}^{\infty} \bigcup_{j=1}^{\infty} \bigcap\left\{W_{l^{-1}, i, r}: 0<r \leq j^{-1}\right\}, \\
{\left[Y=\bigcup_{l=1}^{\infty} \bigcap_{i=1}^{\infty} \bigcup_{j=1}^{\infty} \bigcap\left\{W_{l, i, r}: 0<r \leq j^{-1}\right\}\right] .}
\end{gathered}
$$


5.2 Lemma. Suppose $1 \leq m \leq n$ are integers, $A \subseteq \mathbf{R}^{n}$ and $\tau_{a}(x)=x-a$ whenever $a, x \in \mathbf{R}^{n}$. Let $Y$ be the set of $(a, T) \in \mathbf{R}^{n} \times \mathbf{G}(n, m)$ such that for every $\epsilon>0$ there exist $\eta>0$ and $\rho>0$ such that

$$
\mathscr{H}^{m}\left(\mathbf{C}(T, z, \epsilon r, \epsilon r) \cap \tau_{a}[A]\right) \geq \eta \boldsymbol{\alpha}(m) r^{m}
$$

for every $0<r \leq \rho$ and for every $z \in T \cap \mathbf{B}(0, r)$.

Then $Y$ is a Borel subset of $\mathbf{R}^{n} \times \mathbf{G}(n, m)$.

Proof. We prove that $\left(\mathbf{R}^{n} \times \mathbf{G}(n, m)\right) \sim Y$ is a Borel subset of $\mathbf{R}^{n} \times \mathbf{G}(n, m)$. For every $\epsilon>0, \eta>0$ and $0<\rho_{2}<\rho_{1}$ suppose $W_{\epsilon, \eta, \rho_{1}, \rho_{2}}$ is the set of $(a, T) \in \mathbf{R}^{n} \times \mathbf{G}(n, m)$ such that

$$
\mathscr{H}^{m}\left(\mathbf{C}(T, z, \epsilon r, \epsilon r) \cap \tau_{a}[A]\right) \leq \eta \boldsymbol{\alpha}(m) r^{m}
$$

for some $z \in \mathbf{B}(0, r) \cap T$ and some $\rho_{2} \leq r \leq \rho_{1}$. We prove that $W_{\epsilon, \eta, \rho_{1}, \rho_{2}}$ is a closed subset of $\mathbf{R}^{n} \times \mathbf{G}(n, m)$. Suppose $\left(a_{j}, T_{j}\right) \in W_{\epsilon, \eta, \rho_{1}, \rho_{2}}, j \geq 1$, is a sequence converging to $(a, T) \in \mathbf{R}^{n} \times \mathbf{G}(n, m)$ as $j \rightarrow \infty$. Therefore there exist sequences $\rho_{2} \leq r_{j} \leq \rho_{1}$ and $z_{j} \in T_{j} \cap \mathbf{B}\left(0, r_{j}\right)$, for $j \geq 1$, such that

$$
\mathscr{H}^{m}\left(\mathbf{C}\left(T_{j}, z_{j}, \epsilon r_{j}, \epsilon r_{j}\right) \cap \tau_{a_{j}}[A]\right) \leq \eta \boldsymbol{\alpha}(m) r_{j}^{m} \quad \text { for every } j \geq 1 .
$$

Then there exist $z \in \mathbf{R}^{n}$ and $r \in \mathbf{R}$ such that, possibly passing to a subsequence, $z_{j} \rightarrow z$ and $r_{j} \rightarrow r$ as $j \rightarrow \infty$. Observe that $z \in \mathbf{B}(0, r) \cap T$ and $\rho_{2} \leq r \leq \rho_{1}$. For each $j \geq 1$ we define

$$
B_{j}=\mathbf{C}\left(T_{j}, z_{j}, \epsilon r_{j}, \epsilon r_{j}\right) \cap \tau_{a_{j}}[A], \quad B=\mathbf{C}(T, z, \epsilon r, \epsilon r) \cap \tau_{a}[A],
$$

and one may easily verify that

$$
B \subseteq \bigcup_{h=1} \bigcap_{k=h}^{\infty} \tau_{a-a_{k}}\left[B_{k}\right]
$$

Now we can use [Fed69, 2.1.5(1)] to conclude that

$$
\mathscr{H}^{m}(B) \leq \liminf _{h \rightarrow \infty} \mathscr{H}^{m}\left(\tau_{a-a_{h}}\left[B_{h}\right]\right) \leq \boldsymbol{\alpha}(m) \eta r^{m} .
$$

Therefore $(a, T) \in W_{\epsilon, \eta, \rho_{1}, \rho_{2}}$ and $W_{\epsilon, \eta, \rho_{1}, \rho_{2}}$ is a closed subset of $\mathbf{R}^{n} \times \mathbf{G}(n, m)$. If $E \subseteq \mathbf{R}$ is a countable set such that $\inf E=0 \notin E$ then it is not difficult to see that

$$
\left(\mathbf{R}^{n} \times \mathbf{G}(n, m)\right) \sim Y=\bigcup_{\epsilon \in E} \bigcap_{\eta \in E} \bigcap_{\rho_{1} \in E} \bigcup_{\rho_{2} \in E} W_{\epsilon, \eta, \rho_{1}, \rho_{2}} .
$$

5.3 Definition. A measure $\phi$ over $X$ is called $\sigma$ finite if there exists a sequence $X_{i}$ such that $\phi\left(X_{i}\right)<\infty$ for every $i \geq 1$ and $X=\bigcup_{i=1}^{\infty} X_{i}$.

5.4 Theorem. Suppose $1 \leq m \leq n$ and $k \geq 1$ are integers, $0 \leq \alpha \leq 1, A \subseteq \mathbf{R}^{n}$ such that $\mathscr{H}^{m}\llcorner A$ is $\sigma$ finite and for every $a \in A$ there exists an $m$ dimensional submanifold $B \subseteq \mathbf{R}^{n}$ of class $(k, \alpha)$ such that $a \in B$ and the following condition $(*)$ is satisfied. For every $\epsilon>0$

$$
\lim _{r \rightarrow 0} \frac{\mathscr{H}^{m}\left(A \cap \mathbf{B}(a, r) \cap\left\{z: \boldsymbol{\delta}_{B}(z)>\epsilon r^{k}\right\}\right)}{\boldsymbol{\alpha}(m) r^{m}}=0
$$


and, if $\alpha>0$, there exists $0 \leq \lambda<\infty$ such that

$$
\lim _{r \rightarrow 0} \frac{\mathscr{H}^{m}\left(A \cap \mathbf{B}(a, r) \cap\left\{z: \boldsymbol{\delta}_{B}(z)>\lambda r^{k+\alpha}\right\}\right)}{\boldsymbol{\alpha}(m) r^{m}}=0 .
$$

Then $A$ is countably $\left(\mathscr{H}^{m}, m\right)$ rectifiable of class $(k, \alpha)$.

Proof. Clearly, we can assume $\mathscr{H}^{m}(A)<\infty$. If $X$ is the set of points $a \in \mathbf{R}^{n}$ such that there exists an $m$ dimensional submanifold $B$ of class $(k, \alpha)$ such that $a \in B$ and (*) is satisfied, then $X$ is an $\mathscr{H}^{m}$ measurable subset of $\mathbf{R}^{n}$ by 3.11 , 5.1 and [Fed69, 2.2.13]. If $E \subseteq \mathbf{R}^{n}$ is an $\mathscr{H}^{m}$ hull of $A$, by [Fed69, 2.1.5(2)], then $A \subseteq E \cap X$ and $E \cap X$ is a $\mathscr{H}^{m}$ measurable subset of $\mathbf{R}^{n}$ that satifies the same hypothesis $A$ does. Therefore we can assume $A$ to be $\mathscr{H}^{m}$ measurable.

If $a \in A$ and $B$ is an $m$ dimensional submanifold of class 1 such that $a \in B$ and $(*)$ is satisfied then, by 3.11 and 3.6 , we get that

$$
\operatorname{Tan}^{* m}\left(\mathscr{H}^{m}\llcorner A, a) \subseteq \operatorname{Tan}(B, a), \quad \boldsymbol{\Theta}^{m}\left(\mathscr{H}^{m}\llcorner A \sim \mathbf{X}(a, \operatorname{Tan}(B, a), \epsilon), a)=0,\right.\right.
$$

for every $\epsilon>0$. Therefore by [Fed69, 2.10.19(2), 3.3.17, 3.2.29] we conclude that $A$ is $\left(\mathscr{H}^{m}, m\right)$ rectifiable of class 1 .

Let $S \in \mathbf{G}(n, m)$, let $U \subseteq S$ be relatively open, let $f: U \rightarrow S^{\perp}$ be a function of class $1, M=\{\chi+f(\chi): \chi \in U\}$, Lip $f<\infty$ and $\mathscr{H}^{m}(M)<\infty$. We prove that $A \cap M$ is $\left(\mathscr{H}^{m}, m\right)$ rectifiable of class $(k, \alpha)$. This evidently implies that $A$ is $\left(\mathscr{H}^{m}, m\right)$ rectifiable of class $(k, \alpha)$. Let $Y$ be the set of points $a \in A \cap M$ such that $\Theta^{m}\left(\mathscr{H}^{m}\llcorner M \sim A, a)=0\right.$. We use 4.2, 4.10 and 3.5 to conclude that

$$
\operatorname{Tan}^{* m}\left(\mathscr{H}^{m}\llcorner A \cap M, a)=\operatorname{Tan}(M, a) \text { for every } a \in Y\right. \text {. }
$$

By Fed69, 2.10.19(4)] we have $\mathscr{H}^{m}(A \cap M \sim Y)=0$. Let

$$
C=S \cap\{\chi: \chi+f(\chi) \in A \cap M\}, \quad D=S \cap\{\chi: \chi+f(\chi) \in Y\},
$$

we observe that $\mathscr{H}^{m}(C \sim D)=0$ and

$$
\Theta^{m}\left(\mathscr{H}^{m}\llcorner S \sim C, \chi)=0 \text { for every } \chi \in D\right. \text {. }
$$

Let $\chi \in D, a=\chi+f(\chi)$ and suppose $B$ is an $m$ dimensional submanifold of class $(k, \alpha)$ such that $a \in B$ and (*) is satisfied. Since $\operatorname{Tan}(B, a) \cap S^{\perp}=\{0\}$, there exist a function $g: S \rightarrow S^{\perp}$ of class $(k, \alpha)$ and an open neighbourhood $V$ of $a$ such that $B \cap V=\{\zeta+g(\zeta): \zeta \in S\} \cap V$. Therefore, by 3.11,

$$
\lim _{r \rightarrow 0} \frac{\mathscr{H}^{m}\left(A \cap \mathbf{B}(a, r) \cap\left\{z:\left|g\left(S_{\natural}(z)\right)-S_{\natural}^{\perp}(z)\right|>\epsilon r^{k}\right\}\right)}{\boldsymbol{\alpha}(m) r^{m}}=0
$$

for every $\epsilon>0$ and, if $\alpha>0$, there exists $0 \leq \lambda<\infty$ such that

$$
\lim _{r \rightarrow 0} \frac{\mathscr{H}^{m}\left(A \cap \mathbf{B}(a, r) \cap\left\{z:\left|g\left(S_{\natural}(z)\right)-S_{\natural}^{\perp}(z)\right|>\lambda r^{k+\alpha}\right\}\right)}{\boldsymbol{\alpha}(m) r^{m}}=0 .
$$

Let $P: S \rightarrow S^{\perp}$ be the $k$ jet of $g$ at $\chi$. If $\epsilon>0$ then, possibly replacing $\lambda$ by a larger number if $\alpha>0$, we can choose $\rho>0$ such that

$$
|g(\zeta)-P(\zeta)| \leq \lambda r^{k+\alpha} \text { if } \alpha>0, \quad|g(\zeta)-P(\zeta)| \leq \epsilon r^{k} \text { if } \alpha=0,
$$


for every $\zeta \in \mathbf{B}(\chi, r)$ and $0<r \leq \rho$. Let $\Gamma=\left(1+(\operatorname{Lip} f)^{2}\right)^{1 / 2}, \gamma=\lambda+\Gamma^{k+\alpha} \lambda$ if $\alpha>0$ and observe that, whenever $0<r \leq \rho$,

$$
\begin{aligned}
& C \cap \mathbf{B}(\chi, r) \cap\left\{\zeta:|f(\zeta)-P(\zeta)|>\gamma r^{k+\alpha}\right\} \\
& \quad \subseteq S_{\natural}\left[A \cap M \cap \mathbf{B}(a, \Gamma r) \cap\left\{z:\left|S_{\natural}^{\perp}(z)-g\left(S_{\natural}(z)\right)\right|>\lambda \Gamma^{k+\alpha} r^{k+\alpha}\right\}\right] \quad \text { if } \alpha>0, \\
& C \cap \mathbf{B}(\chi, r) \cap\left\{\zeta:|f(\zeta)-P(\zeta)|>2 \epsilon r^{k}\right\} \\
& \quad \subseteq S_{\natural}\left[A \cap M \cap \mathbf{B}(a, \Gamma r) \cap\left\{z:\left|S_{\natural}^{\perp}(z)-g\left(S_{\natural}(z)\right)\right|>\epsilon r^{k}\right\}\right] \quad \text { if } \alpha=0 .
\end{aligned}
$$

Since $\chi$ is arbitrarily chosen in $D$, we infer by 2.7 and 2.12 that there exist countably many functions $g_{j}: S \rightarrow S^{\perp}$ of class $(k, \alpha)$ such that

$$
\mathscr{H}^{m}\left(C \sim \bigcup_{j=1}^{\infty}\left\{\zeta: f(\zeta)=g_{j}(\zeta)\right\}\right)=0,
$$

whence $A \cap M$ is $\left(\mathscr{H}^{m}, m\right)$ rectifiable of class $(k, \alpha)$.

5.5 Theorem. Suppose $n \geq 1, k \geq 1$ are integers, $0 \leq \alpha \leq 1, A \subseteq \mathbf{R}^{n}$ and $X$ is the set of $a \in \mathbf{R}^{n}$ where $A$ is approximately differentiable of order $(k, \alpha)$.

Then $X$ is a Borel subset of $\mathbf{R}^{n}$. Moreover $\operatorname{ap} \operatorname{Tan}(A, \cdot)_{\natural}$ is a Borel map whose domain is a Borel subset of $\mathbf{R}^{n}$ and the same conclusion is true for ap $\mathrm{D}^{j} A$ for every $j \geq 2$.

Proof. First apply 5.1 and 5.2 to see that the set $Z$ of

$$
\left(a, T, \phi_{0}, \ldots, \phi_{k}\right) \in \bigcup_{m=1}^{n}\left[\mathbf{R}^{n} \times \mathbf{G}(n, m) \times \prod_{i=0}^{k} \bigodot^{i}\left(\mathbf{R}^{n}, \mathbf{R}^{n}\right)\right]
$$

such that $\phi_{1}=0$ and the conditions listed in 5.1 and 5.2 are satisfied for every $\lambda>0$ if $\alpha=0$ and for some $0 \leq \lambda<\infty$ if $\alpha>0$, is a Borel set; then use 3.11 and 3.14 to conclude that $Z$ is the graph of a function $f$ mapping $X$ into $\bigcup_{m=1}^{n}\left[\mathbf{G}(n, m) \times \prod_{i=0}^{k} \bigodot^{i}\left(\mathbf{R}^{n}, \mathbf{R}^{n}\right)\right]$; finally apply [Men16, 4.1] to infer that $X$ is a Borel subset of $\mathbf{R}^{n}$ and $f$ is a Borel function. In case $\alpha=0$, this clearly proves the second part of the conclusion.

5.6 Theorem. Suppose $1 \leq m \leq n$ and $k \geq 1$ are integers, $0 \leq \alpha \leq 1, A \subseteq \mathbf{R}^{n}$ is $\mathscr{H}^{m}$ measurable with $\mathscr{H}^{m}(A)<\infty$ and $X$ is the set of $a \in \mathbf{R}^{n}$ such that $A$ is approximately differentiable of order $(k, \alpha)$ at a with $\operatorname{dim} \operatorname{ap} \operatorname{Tan}(A, a)=m$.

Then $X$ is $\left(\mathscr{H}^{m}, m\right)$ rectifiable of class $(k, \alpha)$ and $\mathscr{H}^{m}(X \sim A)=0$.

Proof. Apply 5.4 to get that $A \cap X$ is $\left(\mathscr{H}^{m}, m\right)$ rectifiable of class $(k, \alpha)$. Since $\Theta_{*}^{m}\left(\mathscr{H}^{m}\llcorner A, x)>0\right.$ for every $x \in X$ by [3.14] we infer that $\mathscr{H}^{m}(X \sim A)=0$ by [Fed69, 2.10.19(4)].

5.7 Remark. The pattern of this section follows [Men16, §5].

\section{References}

[Alb94] Giovanni Alberti. On the structure of singular sets of convex functions. Calc. Var. Partial Differential Equations, 2(1):17-27, 1994.

[All72] William K. Allard. On the first variation of a varifold. Ann. of Math. (2), 95:417-491, 1972. 
[AS94] Gabriele Anzellotti and Raul Serapioni. $\mathscr{C}^{k}$-rectifiable sets. J. Reine Angew. Math., 453:1-20, 1994.

[BHS05] Bogdan Bojarski, Piotr Hajłasz, and Paweł Strzelecki. Sard's theorem for mappings in Hölder and Sobolev spaces. Manuscripta Math., 118(3):383-397, 2005.

[Cam64] S. Campanato. Proprietà di una famiglia di spazi funzionali. Ann. Scuola Norm. Sup. Pisa (3), 18:137-160, 1964.

[Del12] Silvano Delladio. Functions of class $C^{1}$ subject to a Legendre condition in an enhanced density set. Rev. Mat. Iberoam., 28(1):127-140, 2012.

[Fed59] Herbert Federer. Curvature measures. Trans. Amer. Math. Soc., 93:418-491, 1959.

[Fed69] Herbert Federer. Geometric measure theory. Die Grundlehren der mathematischen Wissenschaften, Band 153. Springer-Verlag New York Inc., New York, 1969.

[FM99] Ilaria Fragalà and Carlo Mantegazza. On some notions of tangent space to a measure. Proc. Roy. Soc. Edinburgh Sect. A, 129(2):331-342, 1999.

[Isa87] N. M. Isakov. On a global property of approximately differentiable functions. Mat. Zametki, 41(4):500-508, 620, 1987.

[Koh77] Robert V. Kohn. An example concerning approximate differentiation. Indiana Univ. Math. J., 26(2):393-397, 1977.

[Kol16] S. Kolasiński. Higher order rectifiability of measures via averaged discrete curvatures. ArXiv e-prints, pages 1-20, April 2016.

[Mat95] Pertti Mattila. Geometry of sets and measures in Euclidean spaces, volume 44 of Cambridge Studies in Advanced Mathematics. Cambridge University Press, Cambridge, 1995. Fractals and rectifiability.

[Men13] U. Menne. Second order rectifiability of integral varifolds of locally bounded first variation. J. Geom. Anal., 23(2):709-763, 2013.

[Men16] U. Menne. Pointwise differentiability of higher order for sets. ArXiv e-prints, pages 1-33, March 2016.

[MS17] U. Menne and M. Santilli. A geometric second-order-rectifiable stratification for closed subsets of Euclidean space. ArXiv e-prints, March 2017.

[Sim83] Leon Simon. Lectures on geometric measure theory, volume 3 of Proceedings of the Centre for Mathematical Analysis, Australian National University. Australian National University, Centre for Mathematical Analysis, Canberra, 1983.

[Whi51] Hassler Whitney. On totally differentiable and smooth functions. Pacific J. Math., 1:143-159, 1951. 
Max Planck Institute for Gravitational Physics (Albert Einstein Institute)

Am Mühlenberg 1,

D-14476 Golm, Germany

mario.santilli@aei.mpg.de

University of Potsdam, Institute for Mathematics,

OT Golm, Karl-Liebknecht-Straße 24-25,

D-14476 Potsdam, Germany 